\title{
DOUBLING RATIONAL NORMAL CURVES
}

\author{
ROBERTO NOTARI, IGNACIO OJEDA, MARIA LUISA SPREAFICO
}

\begin{abstract}
In this paper, we study double structures supported on rational normal curves. After recalling the general construction of double structures supported on a smooth curve described in [11, we specialize it to double structures on rational normal curves. To every double structure we associate a triple of integers $(2 r, g, n)$ where $r$ is the degree of the support, $n \geq r$ is the dimension of the projective space containing the double curve, and $g$ is the arithmetic genus of the double curve. We compute also some numerical invariants of the constructed curves, and we show that the family of double structures with a given triple $(2 r, g, n)$ is irreducible. Furthermore, we prove that the general double curve in the families associated to $(2 r, r+1, r)$ and $(2 r, 1,2 r-1)$ is arithmetically Gorenstein. Finally, we prove that the closure of the locus containing double conics of genus $g \leq-2$ form an irreducible component of the corresponding Hilbert scheme, and that the general double conic is a smooth point of that component. Moreover, we prove that the general double conic in $\mathbb{P}^{3}$ of arbitrary genus is a smooth point of the corresponding Hilbert scheme.
\end{abstract}

\section{INTRODUCTION}

Non-reduced projective curves arise naturally when one tries to classify smooth curves, where a projective curve is a dimension 1 projective scheme without embedded or isolated 0 -dimensional components. In fact, two of the main tools to classify projective curves are liaison theory and deformation theory.

Given two curves $C$ and $D$ embedded in the projective space $\mathbb{P}^{n}$, we say that they are geometrically linked if they have no common component and their union is an arithmetically Gorenstein curve. More than the geometric links, a modern treatment of the theory takes as its base the algebraic link where two curves are algebraically linked via the arithmetically Gorenstein curve $X$ if $I_{X}: I_{C}=I_{D}$ and $I_{X}: I_{D}=I_{C}$, where $I_{C}, I_{D}, I_{X}$ are the saturated ideals that define the curves $C, D, X$, respectively, in the projective space $\mathbb{P}^{n}$. If $C$ and $D$ have no common irreducible component, the two definitions agree. Liaison theory and even liaison theory are the study of the equivalence classes of the equivalence relation generated by the direct link, and by an even number of direct links, respectively. In $\mathbb{P}^{3}$, a curve is arithmetically Gorenstein if, and only if, it is the complete intersection of two algebraic surfaces. A pioneer in the study of this theory for curves in $\mathbb{P}^{3}$ was F. Gaeta (see [13]). In the quoted paper, he proved that every arithmetically Cohen-Macaulay curve in $\mathbb{P}^{3}$ is in the equivalence class of a line. More in general, every curve sits in an equivalence class, and, for curves in $\mathbb{P}^{3}$, it is known that every curve in a biliaison class can be obtained from the curves of minimal degree in the class via a rather explicit algorithm. This property is known as Lazarsfeld-Rao property ([23], Definition

2000 Mathematics Subject Classification. 14H45, 14C05, 14M05.

Key words and phrases. double structure, arithmetically Gorenstein curves, Hilbert scheme. 
5.4.2), and it was proved in ([22], Ch. IV, Theorem 5.1). The existence of minimal curves and their construction is proved in ([22], Ch. IV, Proposition 4.1, and Theorem 4.3). In [1], the authors proved the Lazarsfeld-Rao property for the curves in the same biliaison class, without the explicit construction of the minimal curves. The minimal arithmetically Cohen-Macaulay curves are the lines. Hence, the Lazarsfeld-Rao property can be seen as a generalization of Gaeta's work. Also if one wants to study smooth curves, the minimal curves in the biliaison class can have quite bad properties, e.g. they can be non-reduced, or they can have a large number of irreducible components. Moreover, the minimal curves in a biliaison class form an irreducible family of curves with fixed degree and arithmetic genus. Today, it is not known if the equivalence classes of curves in $\mathbb{P}^{n}$ have the same properties as those in $\mathbb{P}^{3}$ (see [25], 8], [16] for evidence both ways).

To study the properties of smooth curves, one can also try to deform the smooth curve to a limit curve and investigate the properties one is interested in on the limit curve. If those properties are shared by the limit curve and the deformation behaves well with respect to the considered properties, then the general curve shares the same properties of the limit curve. Often, the limit curves are non-reduced curves. In the papers [12], [3], [10], the authors study Green's conjecture concerning the free resolution of a canonical curve by reducing it to the study of a similar conjecture for double structures on $\mathbb{P}^{1}$ called ribbons.

Both described approaches lead to the study of families of curves. The universal family of curves of fixed degree $d$ and arithmetic genus $g$ is the Hilbert scheme $\mathcal{H} i l b_{d t+1-g}\left(\mathbb{P}^{n}\right)$, where, for us, $\mathcal{H} i l b_{d t+1-g}\left(\mathbb{P}^{n}\right)$ is the open locus of the full Hilbert scheme corresponding to locally Cohen-Macaulay 1-dimensional schemes, i.e. corresponding to curves. Since A. Grothendieck proved its existence in [14], the study of the properties of the Hilbert scheme attracted many researchers. In spite of their efforts, only a few properties are known, such as the connectedness of the full Hilbert scheme proved by R. Hartshorne in [15. A current trend of research tries to generalize Hartshorne's result on connectedness to the Hilbert scheme of curves. For partial results on the problem, see, for example, [18], [27. In studying Hilbert schemes, a chance is to relate the local properties of a point on the Hilbert scheme, e.g. its smoothness on $\mathcal{H} i l b_{d t+1-g}\left(\mathbb{P}^{n}\right)$, and the global properties of the curve embedded in $\mathbb{P}^{n}$. With abuse of notation, we denote $C$ both the curve in $\mathbb{P}^{n}$ and the corresponding point on $\mathcal{H} i l b_{d t+1-g}\left(\mathbb{P}^{n}\right)$. It is well known that the tangent space to $\mathcal{H} i l b_{d t+1-g}\left(\mathbb{P}^{n}\right)$ at a point $C$ can be identified with $H^{0}\left(C, \mathcal{N}_{C}\right)$ where $\mathcal{N}_{C}$ is the normal sheaf of the curve $C$ as subscheme of $\mathbb{P}^{n}\left([28]\right.$, Theorem 4.3.5). However, both $\mathcal{N}_{C}$ and its degree 0 global sections are far from being well understood for an arbitrary curve $C$.

Both in liaison and biliaison theory, and in deformation theory, one has often to consider non-reduced curves. The first general construction for non-reduced curves was given by D. Ferrand in [11], where the author constructs a double structure on a smooth curve $C \subset \mathbb{P}^{n}$. The construction was investigated in [6], and generalized in [4] to multiple structures on a smooth support. In the last quoted paper, the authors present a filtration of a multiple structure $X$ on a smooth support $C$ via multiple structures with smaller multiplicity on the same curve $C$. Moreover, they relate the properties of $X$ to the ones of the curves in the filtration. A different filtration was proposed in [20]. When the first multiple structure in either filtration has multiplicity 2 at every point, then it 
comes from Ferrand's construction. In this sense, double structures are the first step in studying multiple structures on a smooth curve $C$. Because of the previous discussion, it is interesting to understand if double structures on curves form irreducible families, if they fill irreducible components of the Hilbert scheme (if so, they cannot be limit curves of smooth curves), and if, among them, there are curves with properties that are preserved under generalization, such as the property of being arithmetically Gorenstein. In the papers [25], 24], [26], the authors study the stated problems for double structures on lines, and more generally for a multiple structure $X$ on a line $L \subset \mathbb{P}^{n}$ satisfying the condition $I_{L}^{2} \subseteq I_{X} \subseteq I_{L}$, called ropes in the literature. In the present paper, we address the same problems for double structures supported on the most natural generalization of a line, i.e. a rational normal curve. In [21], the author considers double structures on rational normal curves, but he is interested in the ones with linear resolution, a class of curves different from the ones we investigate.

The plan of the paper is the following. In section 2, we recall Ferrand's construction of double structures on smooth curves, and we specialize it to construct double structures on rational normal curves. To set notation and for further use in the paper, we recall some known facts about rational normal curves. Moreover, we prove that we obtain the saturated ideal of the double structure directly from the construction, and we compute the Hilbert polynomial (and hence the arithmetic genus) of the double structure in terms of the numerical data of the construction. Finally, we compute the dimension of the irreducible family of double rational normal curves of given genus. In section 3, we compute the Hartshorne-Rao function $h^{1} \mathcal{I}_{X}(j)$ of such a doubling $X$, for $j \neq 2$, and we bound $h^{1} \mathcal{I}_{X}(2)$. To get the results, we give also some results about the ideal sheaf $\mathcal{I}_{C}^{2}$ where $C=X_{\text {red }}$ is the rational normal curve support of $X$. Probably, the results we prove on $\mathcal{I}_{C}^{2}$ are folklore, but we did not find references in literature. In section 4 , we prove that, among the double curves we are studying, we can obtain arithmetically Gorenstein curves. In more detail, it happens in two cases: if $X$ has genus $r+1$ in $\mathbb{P}^{r}$, i.e. $X$ has degree and genus of a canonical curve in $\mathbb{P}^{r}$, and if $X$ has genus 1 in $\mathbb{P}^{2 r-1}$, i.e. $X$ has degree and genus of a non-degenerate normal elliptic curve in $\mathbb{P}^{2 r-1}$. The former curves were originally studied in [3] to understand Green' s conjecture on the free resolution of canonical curves. In the same paper, the authors, together with J. Harris, prove that the considered double structures on rational normal curves are smooth points of the component of the Hilbert scheme containing canonical curves ([3], Theorem 6.1). In the last section of the paper, we study the local properties of $H(4, g, n)$, and we show that, if $g \leq-2$, then $H(4, g, n)$ is open in a generically smooth irreducible component of the Hilbert scheme $\mathcal{H}_{i l b_{4 t+1-g}}\left(\mathbb{P}^{n}\right)$. Moreover, we also prove that the general double conic is a smooth point of $H(4, g, 3)$ with $g \geq-1$, that $H(4, g, 3)$ is not an irreducible component of $\mathcal{H} i l b_{4 t+1-g}\left(\mathbb{P}^{3}\right)$, and we exhibit the general element of the irreducible component $\overline{H(4, g, 3)}$ containing $H(4, g, 3)$. The results in this section partially complete the ones in [27]. In fact, in [27], the authors prove that the Hilbert scheme $\mathcal{H}_{i l b} b_{4 t+1-g}\left(\mathbb{P}^{3}\right)$ is connected, but do not study its local properties. By the way, in [27], the double conics are studied as particular curves contained in a double plane, curves studied in [18], and so their construction and their properties are not considered. Finally, in [5], the authors proved that double conics in $\mathbb{P}_{\mathbb{C}}^{3}$ of genus -5 are smooth points of the Hilbert scheme $\mathcal{H} i b_{4 t+6}\left(\mathbb{P}_{\mathbb{C}}^{3}\right)$. 
We want to warmly thank the anonymous referee for his/her comments and remarks and N. Manolache for pointing us a misprint in an earlier draft of the paper.

\section{Construction of double Rational normal Curves}

Let $K$ be an algebraically closed field of characteristic 0 and let $\mathbb{P}^{n}$ be the $n$-dimensional projective space over $K$ defined as $\mathbb{P}^{n}=\operatorname{Proj}\left(R:=K\left[x_{0}, \ldots, x_{n}\right]\right)$. If $X \subset \mathbb{P}^{n}$ is a closed subscheme, we define $\mathcal{I}_{X}$ its ideal sheaf in $\mathcal{O}_{\mathbb{P} n}$ and we define the normal sheaf $\mathcal{N}_{X}$ of $X$ in $\mathbb{P}^{n}$ as $\mathcal{N}_{X}=\mathcal{H}_{o m} \mathbb{P}^{n}\left(\mathcal{I}_{X}, \mathcal{O}_{X}\right)=\mathcal{H} o m_{X}\left(\frac{\mathcal{I}_{X}}{\mathcal{I}_{X}^{2}}, \mathcal{O}_{X}\right)$. The saturated ideal of $X$ is the ideal $I_{X}=\oplus_{j \in \mathbb{Z}} H^{0}\left(\mathbb{P}^{n}, \mathcal{I}_{X}(j)\right) \subseteq R$, and it is a homogeneous ideal. The homogeneous coordinate ring of $X$ is defined as $R_{X}=R / I_{X}$, and it is naturally graded over $\mathbb{Z}$. The Hilbert function of $X$ is then the function defined as $h_{X}(j)=\operatorname{dim}_{K}\left(R_{X}\right)_{j}$, degree $j$ part of $R_{X}$, for $j \in \mathbb{Z}$. Finally, it is known that there exists a polynomial $P(t) \in \mathbb{Q}[t]$, called Hilbert polynomial of $X$, that verifies $P(t)=h_{X}(t)$ for $t \in \mathbb{Z}, t \gg 0$. The degree of $P(t)$ is the dimension of $X$. If $X$ is a locally Cohen-Macaulay curve, then $P(t)=d t+1-g$ for some integers $d, g$, referred to as degree and arithmetic genus of $X$, respectively.

Given a smooth curve $C \subset \mathbb{P}^{n}$, there is a well known method, due to D. Ferrand (see [11]), to construct a non-reduced curve $X$, having $C$ as support, and multiplicity 2 at each point. $X$ is called a doubling of $C$.

Ferrand's method works as follows.

Let $\mathcal{I}_{C}$ be the ideal sheaf of $C \subset \mathbb{P}^{n}$, and let $\mathcal{I}_{C} / \mathcal{I}_{C}^{2}$ be its conormal sheaf. If $\mathcal{L}$ is a line bundle on $C$, every surjective morphism $\mu: \frac{\mathcal{I}_{C}}{\mathcal{I}_{C}^{2}} \rightarrow \mathcal{L}$ gives a doubling $X$ of $C$ defined by the ideal sheaf $\mathcal{I}_{X}$ such that $\operatorname{ker}(\mu)=\mathcal{I}_{X} / \mathcal{I}_{C}^{2}$. The curves $C, X$ and the line bundle $\mathcal{L}$ are related each other via the exact sequences

$$
0 \rightarrow \mathcal{I}_{X} \rightarrow \mathcal{I}_{C} \rightarrow \mathcal{L} \rightarrow 0
$$

and

$$
0 \rightarrow \mathcal{L} \rightarrow \mathcal{O}_{X} \rightarrow \mathcal{O}_{C} \rightarrow 0
$$

Moreover, $X$ is a locally Cohen-Macaulay curve and its dualizing sheaf satisfies $\omega_{X} \mid C=$ $\mathcal{L}^{-1}$.

We are interested in studying doublings of rational normal curves, where, for us, a rational normal curve $C$ of degree $r$ is the image of

$$
\mathbb{P}^{1} \stackrel{v_{r}}{\longrightarrow} \mathbb{P}^{r} \longrightarrow \mathbb{P}^{n}
$$

where $v_{r}$ is the Veronese embedding and the second map is a linear embedding of $\mathbb{P}^{r}$ in $\mathbb{P}^{n}$ with $r \leq n$. To make effective Ferrand's construction in our case, we recall some known results about rational normal curves, and fix some notation.

Let $\mathbb{P}^{r} \cong L=V\left(x_{r+1}, \ldots, x_{n}\right) \subseteq \mathbb{P}^{n}=\operatorname{Proj}\left(R:=K\left[x_{0}, \ldots, x_{n}\right]\right)$ and let $C \subset L$ be the rational normal curve defined by the $2 \times 2$ minors of the matrix

$$
A=\left(\begin{array}{cccc}
x_{0} & x_{1} & \ldots & x_{r-1} \\
x_{1} & x_{2} & \ldots & x_{r}
\end{array}\right)
$$


In $L$ the resolution of the saturated ideal $I_{C, L} \subset S:=K\left[x_{0}, \ldots, x_{r}\right]$ of $C$ is described by the Eagon-Northcott complex and it is

$$
\begin{aligned}
0 \rightarrow & \wedge^{r} F \otimes S_{r-2}(G)^{*} \otimes \wedge^{2} G^{*} \rightarrow \wedge^{r-1} F \otimes S_{r-3}(G)^{*} \otimes \wedge^{2} G^{*} \rightarrow \ldots \\
& \ldots \rightarrow \wedge^{3} F \otimes S_{1}(G)^{*} \otimes \wedge^{2} G^{*} \rightarrow \wedge^{2} F \otimes S_{0}(G)^{*} \otimes \wedge^{2} G^{*} \rightarrow \wedge^{0} F \otimes S_{0}(G) \rightarrow 0
\end{aligned}
$$

where $F=S^{r}(-1), G=S^{2}$ and $\varphi_{A}: F \rightarrow G$ is defined by the matrix $A$.

Remark 2.1. Because of the definition of $F$ and $G$ we have that the complex ends as follows

$$
\cdots \rightarrow \wedge^{3} F \otimes G^{*} \stackrel{\varepsilon}{\longrightarrow} \wedge^{2} F \stackrel{\phi_{A}}{\longrightarrow} S \rightarrow S / I_{C, L} \rightarrow 0
$$

where $\phi_{A}$ is defined via the $2 \times 2$ minors of $A$.

Let $e_{1}, \ldots, e_{r}$ be the canonical basis of $F$ and let $f_{1}, f_{2}$ be the canonical basis of $G^{*}$. Then, the map $\varepsilon$ is defined as

$$
\varepsilon\left(e_{i} \wedge e_{j} \wedge e_{h} \otimes f_{k}\right)=x_{i-2+k} e_{j} \wedge e_{h}-x_{j-2+k} e_{i} \wedge e_{h}+x_{h-2+k} e_{i} \wedge e_{j}
$$

for every $1 \leq i<j<h \leq r, k=1,2$ (e.g. see A2.6.1 in [9]).

Now, we compute the resolution of the saturated ideal $I_{C} \subset R$ of $C$. Of course, $I_{C}=$ $I_{C, L}^{e}+I_{L}=I_{C, L}^{e}+\left\langle x_{r+1}, \ldots, x_{n}\right\rangle$, where $I_{C, L}^{e}$ is the extension of $I_{C, L}$ via the natural inclusion $S \hookrightarrow R$.

To get a minimal free resolution of $I_{C, L}^{e}$ it suffices to tensorise by $\otimes_{S} R$ the minimal free resolution of $I_{C, L}$. To simplify notation, we set $P_{i}=\wedge^{i+1} F \otimes_{S} S_{i-1}(G)^{*} \otimes_{S} \wedge^{2} G^{*} \otimes_{S} R$, for $i=1, \ldots, r-1$. Hence, the minimal free resolution of $I_{C, L}^{e}$ is equal to

$$
0 \rightarrow P_{r-1} \stackrel{\varepsilon_{r-1}}{\longrightarrow} P_{r-2} \stackrel{\varepsilon_{r-2}}{\longrightarrow} \cdots \stackrel{\varepsilon_{3}}{\longrightarrow} P_{2} \stackrel{\varepsilon_{2}}{\longrightarrow} P_{1} \stackrel{\varepsilon_{1}}{\longrightarrow} I_{C, L}^{e} \rightarrow 0,
$$

where the maps are obtained by tensorising the maps of the minimal free resolution of $I_{C, L}$ times the identity of $R$.

The minimal free resolution of $I_{L}$ is given by the Koszul complex over $x_{r+1}, \ldots, x_{n}$. Let $Q=R^{n-r}(-1)$ with canonical basis $e_{r+1}, \ldots, e_{n}$ and let $\delta: Q \rightarrow I_{L}$ be defined as $\delta\left(e_{i}\right)=x_{i}, i=r+1, \ldots, n$. If we set $Q_{i}=\wedge^{i} Q$ then the minimal free resolution of $I_{L}$ is equal to

$$
0 \rightarrow Q_{n-r} \stackrel{\delta_{n-r}}{\longrightarrow} Q_{n-r-1} \stackrel{\delta_{n-r-1}}{\longrightarrow} \cdots \stackrel{\delta_{3}}{\longrightarrow} Q_{2} \stackrel{\delta_{2}}{\longrightarrow} Q_{1} \stackrel{\delta}{\longrightarrow} I_{L} \rightarrow 0
$$

where $\delta_{i}=\wedge^{i} \delta$.

Given the two resolutions (3) and (44) above, we can compute their tensor product (for the definition and details, see [9], §17.3), and we get

$$
0 \rightarrow N_{n-1} \longrightarrow N_{n-2} \longrightarrow \ldots \longrightarrow N_{2} \longrightarrow N_{1}
$$

where $N_{i}=\oplus_{j+k=i+1} P_{j} \otimes_{R} Q_{k}$.

Lemma 2.2. The complex (5) is a minimal free resolution of $I_{C, L}^{e} \cap I_{L}$.

Proof. The free module $N_{i}$ is isomorphic to $R^{\beta_{i}}(-i-2)$ with $\beta_{i}=\sum_{j+k=i+1} j\left(\begin{array}{c}r \\ j+1\end{array}\right)\left(\begin{array}{c}n-r \\ k\end{array}\right)$, as computed from its definition. Hence, no addendum can be canceled because of the shifts. It follows that if the complex (5) is a resolution of $I_{C, L}^{e} \cap I_{L}$ then it is its minimal free resolution. 
At first, we prove that $I_{C, L}^{e} \cap\left\langle x_{r+1}, \ldots, x_{l}\right\rangle=I_{C, L}^{e} \cdot\left\langle x_{r+1}, \ldots, x_{l}\right\rangle$ for every $l=r+$ $1, \ldots, n$. In fact, if $f \in I_{C, L}^{e} \cap\left\langle x_{r+1}, \ldots, x_{l}\right\rangle$ and $I_{C, L}^{e}=\left\langle g_{1}, \ldots, g_{t}\right\rangle$, then there exist $h_{1}, \ldots, h_{t} \in R$ such that $f=h_{1} g_{1}+\ldots h_{t} g_{t} \in\left\langle x_{r+1}, \ldots, x_{l}\right\rangle$. For each $i=1, \ldots, t$ there exist $h_{i}^{\prime} \in K\left[x_{0}, \ldots, x_{r}, x_{l+1}, \ldots, x_{n}\right]$ and $h_{i}^{\prime \prime} \in\left\langle x_{r+1}, \ldots, x_{l}\right\rangle$, both unique, such that $h_{i}=h_{i}^{\prime}+h_{i}^{\prime \prime}$. Hence, $h_{1}^{\prime} g_{1}+\cdots+h_{t}^{\prime} g_{t} \in\left\langle x_{r+1}, \ldots, x_{l}\right\rangle$ and so it is equal to 0 , because the variables $x_{r+1}, \ldots, x_{l}$ appear neither in the $g_{i}$ 's nor in the $h_{j}^{\prime}$ 's. Then, we have $f=h_{1}^{\prime \prime} g_{1}+\cdots+h_{t}^{\prime \prime} g_{t} \in I_{C, L}^{e} \cdot\left\langle x_{r+1}, \ldots, x_{l}\right\rangle$.

To prove that the complex (5) is a resolution of $I_{C, L}^{e} \cap I_{L}$ we use induction on the number of generators of $I_{L}$.

If $I_{L}=\left\langle x_{r+1}\right\rangle$, then $I_{C, L}^{e}(-1) \stackrel{x_{r+1}}{\longrightarrow} I_{C, L}^{e} \cdot I_{L}$ is a degree 0 isomorphism, and so the resolution of $I_{C, L}^{e} \cdot I_{L}$ is the one of $I_{C, L}^{e}$ shifted by -1 . In this case, $0 \rightarrow R(-1) \stackrel{\cdot x_{r+1}}{\longrightarrow} I_{L} \rightarrow 0$ is the resolution of $I_{L}$. Thus, the complex (5) is equal to (3) tensorised by $\otimes_{R} R(-1)$ and so it is the resolution of $I_{C, L}^{e} \cdot I_{L}$.

Assume now that $I_{L}=\left\langle x_{r+1}, \ldots, x_{n}\right\rangle$ and that the statement holds for $I_{L}^{\prime}=\left\langle x_{r+1}, \ldots\right.$, $\left.x_{n-1}\right\rangle$.

The two ideals $I_{C, L}^{e} \cdot I_{L}$ and $I_{C, L}^{e} \cdot I_{L}^{\prime}+I_{C, L}^{e} \cdot\left\langle x_{n}\right\rangle$ are equal. Moreover, $I_{C, L}^{e} \cdot I_{L}^{\prime} \cap I_{C, L}^{e} \cdot\left\langle x_{n}\right\rangle$ is equal to $I_{C, L}^{e} \cdot I_{L}^{\prime} \cdot\left\langle x_{n}\right\rangle \cong I_{C, L}^{e} \cdot I_{L}^{\prime}(-1)$. In fact, let $f \in I_{C, L}^{e}$ and assume that $x_{n} f \in I_{C, L}^{e} \cdot I_{l}^{\prime}$. Then, $x_{n} f \in I_{C, L}^{e}$ and $x_{n} f \in I_{L}^{\prime}$. But both the ideals are prime and $x_{n}$ belongs neither to $I_{C, L}^{e}$ nor to $I_{L}^{\prime}$. Hence, $f \in I_{C, L}^{e} \cap I_{L}^{\prime}=I_{C, L} \cdot I_{L}^{\prime}$ and the statement follows because the converse inclusion is evident. We have then the following short exact sequence

$$
0 \rightarrow I_{C, L}^{e} \cdot I_{L}^{\prime}(-1) \longrightarrow I_{C, L}^{e} \cdot I_{L}^{\prime} \oplus I_{C, L}^{e} \cdot\left\langle x_{n}\right\rangle \longrightarrow I_{C, L}^{e} \cdot I_{L} \rightarrow 0
$$

and the claim follows by applying the mapping cone procedure.

Proposition 2.3. With the same notation as before, the minimal free resolution of $I_{C}$ is

$$
0 \rightarrow N_{n-1} \stackrel{\varepsilon_{n-1}^{\prime}}{\longrightarrow} \cdots \stackrel{\varepsilon_{3}^{\prime}}{\longrightarrow} P_{2} \oplus Q_{2} \oplus N_{1} \stackrel{\varepsilon_{2}^{\prime}}{\longrightarrow} P_{1} \oplus Q_{1} \stackrel{\varepsilon_{1}^{\prime}}{\longrightarrow} I_{C} \rightarrow 0
$$

where $\varepsilon_{2}^{\prime}: N_{1}=P_{1} \otimes Q_{1} \longrightarrow P_{1} \oplus Q_{1}$ is defined as

$$
\left(\begin{array}{ccc}
-x_{r+1} i d_{P_{1}} & \cdots & -x_{n} i d_{P_{1}} \\
\varepsilon_{1} & \cdots & \varepsilon_{1}
\end{array}\right) .
$$

Proof. The ideal $I_{C}$ is equal to $I_{C, L}^{e}+I_{L}$. Hence, we have the short exact sequence

$$
0 \rightarrow I_{C, L}^{e} \cap I_{L} \longrightarrow I_{C, L}^{e} \oplus I_{L} \longrightarrow I_{C} \rightarrow 0 .
$$

By applying the mapping cone procedure, we get a free resolution of $I_{C}$ that has the shape of our claim. The minimality of the resolution follows because a cancelation takes place in the resolution only if a free addendum of $N_{i}$ splits from the map $N_{i} \rightarrow P_{i} \oplus Q_{i}$. This cannot happen because $N_{i} \cong R^{\beta_{i}}(-i-2), P_{i} \cong R^{i\left({ }_{i+1}^{r}\right)}(-i-1)$, and $Q_{i} \cong R^{\left({ }^{n-r}\right)}(-i)$ and so the twists do not allow the splitting of free addenda.

By sheafifying the previous resolutions, we get the minimal resolutions of $\mathcal{I}_{C}$ and $\mathcal{I}_{L}$ over $\mathcal{O}_{\mathbb{P}^{n}}$, and of $\mathcal{I}_{C, L}$ over $\mathcal{O}_{L}$, that we shall use in what follows. As standing notation, the map $\varepsilon_{i}^{\prime}$ of the resolution of $I_{C}$ will become $\tilde{\varepsilon}_{i}^{\prime}$ after sheafifying the resolution, and the same for the other maps. 
As previously explained, to construct a double structure $X$ supported on $C$ we need a surjective morphism $\mu: \frac{\mathcal{I}_{C}}{\mathcal{I}_{C}^{2}} \rightarrow \mathcal{L}$ where $\mathcal{L}$ is an invertible sheaf on $C$. We know that $\mathcal{I}_{C} / \mathcal{I}_{C}^{2} \cong \mathcal{I}_{C} \otimes \mathcal{O}_{C}$ and so, if we tensorise the resolution of $\mathcal{I}_{C}$ with $\mathcal{O}_{C}$ we get $\mathcal{I}_{C} / \mathcal{I}_{C}^{2} \cong$ $\operatorname{coker}\left(\tilde{\varepsilon}_{2}^{\prime} \otimes i d_{\mathcal{O}_{C}}\right)$. Moreover, it is easy to prove the following

Proposition 2.4. $\operatorname{coker}\left(\tilde{\varepsilon}_{2}^{\prime} \otimes i d_{\mathcal{O}_{C}}\right) \cong \operatorname{coker}\left(\tilde{\varepsilon}_{2} \otimes i d_{\mathcal{O}_{C}}\right) \oplus \mathcal{O}_{C}^{n-r}(-1)$.

Proof. The restrictions of $\tilde{\varepsilon}_{2}^{\prime} \otimes i d_{\mathcal{O}_{C}}$ to $\tilde{N}_{1}$ and to $\tilde{Q}_{2}$ are the null maps because the entries of the mentioned restrictions belong to $I_{C}$.

The curve $C$ is isomorphic to $\mathbb{P}^{1}$, and an isomorphism $j: \mathbb{P}^{1} \rightarrow C$ is defined as $j(t$ : $u)=\left(t^{r}: t^{r-1} u: \cdots: u^{r}: 0: \cdots: 0\right)$. We have that $j^{*}\left(\mathcal{O}_{C}^{n-r}(-1)\right)=\mathcal{O}_{\mathbb{P}^{1}}^{n-r}(-r)$ and $j^{*}\left(\operatorname{coker}\left(\tilde{\varepsilon}_{2} \otimes i d_{\mathcal{O}_{C}}\right)\right)=\operatorname{coker}\left(j^{*}\left(\tilde{\varepsilon}_{2} \otimes i d_{\mathcal{O}_{C}}\right)\right) \cong \mathcal{O}_{\mathbb{P}^{1}}^{r-1}(-r-2)$ (see Lemma 5.4 in [3] for the last isomorphism). Hence, on $\mathbb{P}^{1}$, the conormal sheaf of $C \subseteq \mathbb{P}^{n}$ is isomorphic to $\mathcal{O}_{\mathbb{P}^{1}}^{r-1}(-r-2) \oplus \mathcal{O}_{\mathbb{P}^{1}}^{n-r}(-r)$.

Now, we make some effort to explicitly write the previous isomorphism.

Lemma 2.5. Let $e_{1}, \ldots, e_{r}$ and $g_{1}, \ldots, g_{r-1}$ be the canonical bases of $\mathcal{O}_{\mathbb{P} 1}^{r}(-r)$ and of $\mathcal{O}_{\mathbb{P}^{1}}^{r-1}(-r-2)$, respectively, and let $\psi_{r}: \wedge^{2} \mathcal{O}_{\mathbb{P}^{1}}^{r}(-r) \longrightarrow \mathcal{O}_{\mathbb{P}^{1}}^{r-1}(-r-2)$ be defined as

$$
\psi_{r}\left(e_{p} \wedge e_{q}\right)=\sum_{h=p}^{q-1} t^{r-h-1} u^{h-1} g_{p+q-1-h}, \quad \text { for every } 1 \leq p<q \leq r .
$$

Then, $\psi_{r}$ is surjective and $\operatorname{ker}\left(\psi_{r}\right) \cong \mathcal{O}_{\mathbb{P}^{1}}^{\left(\begin{array}{r}2-1 \\ 2\end{array}\right)}(-2 r-2)$.

Proof. The map $\psi_{r}$ is surjective. In fact, $\psi_{r}\left(e_{1} \wedge e_{2}\right), \ldots, \psi_{r}\left(e_{1} \wedge e_{r}\right)$ are linearly independent at each point of $\mathbb{P}^{1}$ except $(0: 1)$, while $\psi_{r}\left(e_{1} \wedge e_{r}\right), \ldots, \psi_{r}\left(e_{r-1} \wedge e_{r}\right)$ are linearly independent at each point of $\mathbb{P}^{1}$ except $(1: 0)$. Hence, $\psi_{r}$ is surjective at every point of $\mathbb{P}^{1}$ and so it is surjective.

Then, we have the following short exact sequence

$$
0 \rightarrow \operatorname{ker}\left(\psi_{r}\right) \longrightarrow \wedge^{2} \mathcal{O}_{\mathbb{P}^{1}}^{r}(-r) \longrightarrow \mathcal{O}_{\mathbb{P} 1}^{r-1}(-r-2) \rightarrow 0,
$$

where $\operatorname{ker}\left(\psi_{r}\right)$ is a locally free $\mathcal{O}_{\mathbb{P}^{1}}$-module of rank $\left(\begin{array}{c}r \\ 2\end{array}\right)-(r-1)=\left(\begin{array}{c}r-1 \\ 2\end{array}\right)$. By $([19]$, Proposition 10.5.1), due to Grothendieck,

$$
\operatorname{ker}\left(\psi_{r}\right) \cong \bigoplus_{i=1}^{\left(\begin{array}{c}
r-1 \\
2
\end{array}\right)} \mathcal{O}_{\mathbb{P}^{1}}\left(-2 r-a_{i}\right)
$$

for some integers $0 \leq a_{1} \leq \cdots \leq a_{\left(\begin{array}{c}r-1 \\ 2\end{array}\right)}$ which are uniquely determined by ker $\psi_{r}$.

It is an easy check to prove that

$$
u^{2} e_{p} \wedge e_{q}-t u e_{p} \wedge e_{q+1}-t u e_{p+1} \wedge e_{q}+t^{2} e_{p+1} \wedge e_{q+1} \in \operatorname{ker}\left(\psi_{r}\right)
$$

for $p=1, \ldots, r-2$ and $q=p+1, \ldots, r-1$. Of course, if $q=p+1$, the third addendum is missing. Hence, we have $\left(\begin{array}{c}r-1 \\ 2\end{array}\right)$ linearly independent elements of $\operatorname{ker}\left(\psi_{r}\right)$, and so there is a subsheaf of $\operatorname{ker}\left(\psi_{r}\right)$ that is isomorphic to $\mathcal{O}_{\mathbb{P}^{1}}^{\left(\begin{array}{c}r-1 \\ 2\end{array}\right)}(-2 r-2)$. Hence, the statement holds if we prove that $a_{1}=2$ or equivalently that ker $\psi_{r}$ does not contain elements of the form $\sum_{1 \leq p<q \leq r} l_{p q} e_{p} \wedge e_{q}$ with $\operatorname{deg}\left(l_{p q}\right) \leq 1$. To see this, we proceed by induction on $r$. 
Assume $r=3$. Then, $\psi_{3}$ is represented by the matrix

$$
\left(\begin{array}{lll}
t & u & 0 \\
0 & t & u
\end{array}\right)
$$

The syzygy module is then generated by $u^{2} e_{1} \wedge e_{2}-t u e_{1} \wedge e_{3}+t^{2} e_{2} \wedge e_{3}$, and so the claim holds if $r=3$.

Assume the claim holds for $\psi_{r-1}$. If $\sum_{1 \leq p<q \leq r} l_{p q} e_{p} \wedge e_{q} \in \operatorname{ker}\left(\psi_{r}\right)$ then, for every $j=2, \ldots, r$, the $l_{p q}$ 's satisfy the equation

$$
l_{1 j} t^{r-2}+l_{1, j+1} t^{r-3} u+\cdots+l_{1 r} t^{j-2} u^{r-j}+u p_{j}=0
$$

where $p_{j} \in\left(l_{23}, \ldots, l_{r-1, r}\right)$, and $p_{2}=0$. Then, $l_{1 j} \in(u)$, for every $j=2, \ldots, r$. Furthermore, for $j=2$, we have also that $l_{1 r} \in(t)$. By degree reasons, $l_{1 r}=0$. By substituting in the equation corresponding to $j=2$, we get that $t$ can be canceled and so we get that $l_{1, r-1} \in(t)$. Again by degree reasons, $l_{1, r-1}=0$. By iterating the argument, we get that $l_{1 j}=0$ for $j=2, \ldots, r$. The map $\psi_{r}$ restricted to the span of $e_{2} \wedge e_{3}, \ldots, e_{r-1} \wedge e_{r}$ is the null map on the first addendum of $\mathcal{O}_{\mathbb{P} 1}^{r-1}(-r-2)$ and $u \psi_{r-1}$ on the remaining addenda. But then $\sum_{2 \leq p<q \leq r} l_{p q} e_{p} \wedge e_{q} \in \operatorname{ker}\left(\psi_{r}\right)$ implies $\sum_{2 \leq p<q \leq r} l_{p q} e_{p} \wedge e_{q} \in \operatorname{ker}\left(\psi_{r-1}\right)$, and we conclude by induction assumption.

Theorem 2.6. With the same hypotheses as Lemma 2.5, the sequence

$$
\wedge^{3} \mathcal{O}_{\mathbb{P}^{1}}^{r}(-r) \otimes\left(\mathcal{O}_{\mathbb{P}^{1}}^{2}\right)^{*} \stackrel{j^{*}\left(\tilde{\varepsilon}_{2} \otimes i d_{\mathcal{O}_{C}}\right)}{\longrightarrow} \wedge^{2} \mathcal{O}_{\mathbb{P}^{1}}^{r}(-r) \stackrel{\psi_{r}}{\longrightarrow} \mathcal{O}_{\mathbb{P}^{1}}^{r-1}(-r-2) \rightarrow 0
$$

is exact.

Proof. We have only to prove that $\operatorname{ker}\left(\psi_{r}\right)=\operatorname{Im}\left(j^{*}\left(\tilde{\varepsilon}_{2} \otimes i d_{\mathcal{O}_{C}}\right)\right)$.

To start, we verify that $\psi_{r} \circ j^{*}\left(\tilde{\varepsilon}_{2} \otimes i d_{\mathcal{O}_{C}}\right)=0$.

It is a simple computation and its details are

$$
\begin{aligned}
& \left.\psi_{r}\left(j^{*}\left(\tilde{\varepsilon}_{2} \otimes i d_{\mathcal{O}_{C}}\right)\right)\left(e_{i} \wedge e_{j} \wedge e_{h} \otimes f_{k}\right)\right)= \\
& =t^{r-i+2-k} u^{i-2+k} \psi_{r}\left(e_{j} \wedge e_{h}\right)-t^{r-j+2-k} u^{j-2+k} \psi_{r}\left(e_{i} \wedge e_{h}\right)+t^{r-h+2-k} u^{h-2+k} \psi_{r}\left(e_{i} \wedge e_{j}\right)= \\
& =t^{r-i+2-k} u^{i-2+k}\left(t^{r-h} u^{h-2} g_{j}+\cdots+t^{r-j-1} u^{j-1} g_{h-1}\right)-t^{r-j+2-k} u^{j-2+k}\left(t^{r-h} u^{h-2} g_{i}+\ldots\right. \\
& \left.\cdots+t^{r-i-1} u^{i-1} g_{h-1}\right)+t^{r-h+2-k} u^{h-2+k}\left(t^{r-j} u^{j-2} g_{i}+\cdots+t^{r-i-1} u^{i-1} g_{j-1}\right)=0 .
\end{aligned}
$$

As last step, we must prove that $\operatorname{ker}\left(\psi_{r}\right)=\operatorname{Im}\left(j^{*}\left(\tilde{\varepsilon}_{2} \otimes i d_{\mathcal{O}_{C}}\right)\right)$.

In Lemma 2.5, we proved that

$$
u^{2} e_{p} \wedge e_{q}-t u e_{p} \wedge e_{q+1}-t u e_{p+1} \wedge e_{q}+t^{2} e_{p+1} \wedge e_{q+1}
$$

for $p=1, \ldots, r-2$ and $q=p+1, \ldots, r-1$, generate $\operatorname{ker}\left(\psi_{r}\right)$.

Furthermore, we have the equalities

$$
\begin{aligned}
u j^{*}\left(\tilde{\varepsilon}_{2} \otimes i d_{\mathcal{O}_{C}}\right)\left(e_{p} \wedge e_{q} \wedge e_{q+1} \otimes f_{k}\right)-t j^{*}\left(\tilde{\varepsilon}_{2} \otimes i d_{\mathcal{O}_{C}}\right)\left(e_{p+1} \wedge e_{q} \wedge e_{q+1} \otimes f_{k}\right)= \\
=t^{r-q+1-k} u^{q-2+k}\left(u^{2} e_{p} \wedge e_{q}-t u e_{p} \wedge e_{q+1}-t u e_{p+1} \wedge e_{q}+t^{2} e_{p+1} \wedge e_{q+1}\right)
\end{aligned}
$$

for every admissible $p<q$, and so $\left(\operatorname{ker}\left(\psi_{r}\right)\right)_{P}=\left(\operatorname{Im}\left(j^{*}\left(\tilde{\varepsilon}_{2} \otimes i d_{\mathcal{O}_{C}}\right)\right)_{P}\right)$ at every point $P \in \mathbb{P}^{1} \backslash\{(1: 0),(0: 1)\}$. At $(t: u)=(0: 1)$ the equality of the stalks follows from $j^{*}\left(\tilde{\varepsilon}_{2} \otimes i d_{\mathcal{O}_{C}}\right)\left(e_{p} \wedge e_{q} \wedge e_{r} \otimes f_{2}\right)=e_{p} \wedge e_{q}$ for every $p=1, \ldots, r-2, q=p+1, \ldots, r-1$ and the fact that $\left(\operatorname{ker}\left(\psi_{r}\right)\right)_{(0: 1)}$ is generated by $e_{p} \wedge e_{q}$ with $p=1, \ldots, r-2, q=p+1, \ldots, r-1$. 
Analogously, we get the claim at $(t: u)=(1: 0)$ by computing $j^{*}\left(\tilde{\varepsilon}_{2} \otimes i d_{\mathcal{O}_{C}}\right)\left(e_{1} \wedge e_{p} \wedge\right.$ $\left.e_{q} \otimes f_{1}\right)$.

When there is no confusion, we' ll write $\psi$ instead of $\psi_{r}$.

Of course, thanks to the isomorphism $j$, the map $\mu: \frac{\mathcal{I}_{C}}{\mathcal{I}_{C}^{2}} \rightarrow \mathcal{L}$ can be written also as $\mu: \mathcal{O}_{\mathbb{P}^{1}}^{r-1}(-r-2) \oplus \mathcal{O}_{\mathbb{P}^{1}}^{n-r}(-r) \rightarrow \mathcal{O}_{\mathbb{P}^{1}}(-r-2+a)$ where $j^{*}(\mathcal{L})=\mathcal{O}_{\mathbb{P}^{1}}(-r-2+a)$ for some $a \geq 0$, because the map $\mu$ is surjective.

Now, the construction can be rewritten as an algorithm: choose the map $\mu$, and consider the map

$$
H_{*}^{0}\left(j_{*}(\mu \circ(\psi \oplus i d))\right): H_{*}^{0}\left(C, \wedge^{2} \mathcal{O}_{C}^{r}(-1) \oplus \mathcal{O}_{C}^{n-r}(-1)\right) \longrightarrow H_{*}^{0} \mathcal{L} .
$$

Let $F_{1}$ be a free $H_{*}^{0}\left(C, \mathcal{O}_{C}\right)$-module such that the complex

$$
F_{1} \stackrel{\nu}{\longrightarrow} H_{*}^{0}\left(C, \wedge^{2} \mathcal{O}_{C}^{r}(-1) \oplus \mathcal{O}_{C}^{n-r}(-1)\right) \stackrel{H_{*}^{0}\left(j_{*}(\mu \circ(\psi \oplus i d))\right)}{\longrightarrow} H_{*}^{0} \mathcal{L}
$$

is exact. Let $N$ be a matrix that represents the map $\nu$, and let $M$ be a lifting of $N$ over $R=H_{*}^{0}\left(\mathbb{P}^{n}, \mathcal{O}_{\mathbb{P}^{n}}\right)$, via the canonical surjective map $R \rightarrow R / I_{C}=H_{*}^{0}\left(C, \mathcal{O}_{C}\right)$. The ideal $I_{X}$ of the doubling $X$ is generated by $I_{C}^{2}+\left[I_{C}\right] M$, where $\left[I_{C}\right]$ is a row matrix with entries equal to the generators of $I_{C}$ in the same order used to write $\psi$.

Now, we investigate more deeply the construction. The data we need to construct such a curve $X$ are: $(i)$ a rational normal curve $C$ of degree $r$ in its linear span $L$ embedded in $\mathbb{P}^{n}$, for some $n \geq r$, together with an isomorphism $j: \mathbb{P}^{1} \rightarrow C$; (ii) a surjective map $\mu: \mathcal{O}_{\mathbb{P}^{1}}^{r-1}(-r-2) \oplus \mathcal{O}_{\mathbb{P}^{1}}^{n-r}(-r) \rightarrow \mathcal{O}_{\mathbb{P}^{1}}(-r-2+a)$ for some $a \geq 0$.

Remark 2.7. For $r \geq 3$ and $a \geq 0$ there exists always a surjective map $\mu$, while, for $r=n=2$, there exists a surjective map $\mu$ if, and only if, $a=0$.

Theorem 2.8. Let $X$ and $X^{\prime}$ be double structures on two rational normal curves $C$ and $C^{\prime}$. Then, $X=X^{\prime}$ if, and only if, $C=C^{\prime}$ and the target maps $\mu$ and $\mu^{\prime}$ differ by an automorphism of $\mathcal{O}_{\mathbb{P}^{1}}(-r-2+a)$ after changing $j^{\prime}$ with $j$.

Proof. Assume first that $C=C^{\prime}$ and $j=j^{\prime}$. If $\mu$ and $\mu^{\prime}$ differ by an automorphism of $\mathcal{O}_{\mathbb{P}^{1}}(-r-2+a)$ then the maps $\mu \circ \psi$ and $\mu^{\prime} \circ \psi$ have the same kernel, and so the curves $X$ and $X^{\prime}$ are defined by the same ideal, i.e. they are equal each other.

Conversely, if $X$ and $X^{\prime}$ are defined by the same ideal, then $X_{\text {red }}$ and $X_{\text {red }}^{\prime}$ are the same curve $C$, because the supporting curve is defined by the only minimal prime ideal associated to $I_{X}$. Up to compose $j^{\prime}$ with an isomorphism of $\mathbb{P}^{1}$ we can assume that $j=j^{\prime}$. The claim follows from ([4], (1.1))

We show with an example how to compute the ideal of such a doubling.

Example 2.9. Let $C \subset \mathbb{P}^{3}=\operatorname{Proj}(K[x, y, z, w])$ be the twisted cubic curve whose ideal is $I_{C}=\left(y^{2}-x z, y z-x w, z^{2}-y w\right)$. We want to construct a double structure $X$ on $C$ contained in $\mathbb{P}^{3}$. As explained, if we set $a=1$, we must choose a surjective map

$$
\mu: \mathcal{O}_{\mathbb{P}^{1}}^{2}(-5) \rightarrow \mathcal{O}_{\mathbb{P}^{1}}(-4) .
$$

Set $\mu=(t, u)$. The map $\mu \circ \psi$ is given by $\mu \circ \psi=\left(t^{2}, 2 t u, u^{2}\right)$, while the map $H_{*}^{0}\left(j_{*}(\mu \circ \psi)\right)$ is given by either $(y, 2 z, w)$ or $(x, 2 y, z)$ (the two apparently different maps agree over $C \backslash\{(1: 0: 0: 0),(0: 0: 0: 1)\})$. Of course, to get the two expressions we multiplied 
$\mu \circ \psi$ times $t$ and $u$ so that the entries have degree multiple of $r=3$, and then we used the isomorphism $j$. The free $R / I_{C}-$ module $F_{1}$ that makes exact the complex

$$
F_{1} \rightarrow\left(R / I_{C}\right)^{3}(-2) \rightarrow H_{*}^{0} \mathcal{L}
$$

is $F_{1}=\left(R / I_{C}\right)^{6}(-3)$ and a matrix that represents the map $F_{1} \rightarrow\left(R / I_{C}\right)^{3}(-2)$ is

$$
N=\left(\begin{array}{cccccc}
2 y & 2 z & 2 w & 0 & 0 & 0 \\
-x & -y & -z & y & z & w \\
0 & 0 & 0 & -2 x & -2 y & -2 z
\end{array}\right)
$$

By lifting $N$ to a matrix $M$ over $R$ via $R \rightarrow R / I_{C}$ we get $M=N$, where the entries are polynomials in $R$ and no more equivalence classes in $R / I_{C}$. The double structure $X$ is then defined by

$$
I_{X}=I_{C}^{2}+\left[I_{C}\right] M
$$

Proposition 2.10. Let $C \subseteq \mathbb{P}^{r} \cong L \subset \mathbb{P}^{n}$ be a rational normal curve of degree $r$, and let $j: \mathbb{P}^{1} \rightarrow C$ be an isomorphism. Let $\mu: \mathcal{O}_{\mathbb{P} 1}^{r-1}(-r-2) \oplus \mathcal{O}_{\mathbb{P 1}}^{n-r}(-r) \rightarrow \mathcal{O}_{\mathbb{P}^{1}}(-r-2+a)$ be a surjective map, and let $X$ be the double structure on $C$ associated to $\mu$. Then, the ideal $I_{X}=I_{C}^{2}+\left[I_{C}\right] M$, constructed as explained, is saturated.

Proof. Let $J=I_{X}^{\text {sat }}$. From the inclusion $J \subseteq I_{C}$, it follows that there exists a matrix $M^{\prime}$ such that $J=I_{C}^{2}+\left[I_{C}\right] M^{\prime}$. Let $N, N^{\prime}$ be the images of $M, M^{\prime}$, respectively, when we restrict the last two matrices to $R / I_{C}$. The matrices $N$ and $N^{\prime}$ both present $H_{*}^{0}\left(C, \wedge^{2} \mathcal{O}_{C}^{r}(-1) \oplus\right.$ $\left.\mathcal{O}_{C}^{n-r}(-1)\right) / \operatorname{ker}\left(H_{*}^{0}\left(j_{*}(\mu \circ(\psi \oplus i d))\right)\right)$, and so the columns of $N$ (resp. $\left.N^{\prime}\right)$ are combination of the ones of $N^{\prime}$ (resp. $\left.N\right)$. Hence, $I_{X}=J$ and $I_{X}$ is saturated.

We want to prove some results about families of doublings. Before stating and proving those results, we compute the Hilbert polynomial of a doubling $X$ in terms of the degree of $\mathcal{L}$. Of course, the degree of $X$ is twice the degree of the rational normal curve $C=X_{\text {red }}$ and so we have to compute the genus of $X$.

Proposition 2.11. Let $X$ be a doubling of a degree $r$ rational normal curve $C$ defined by a map $\mu$ as above. Then, the Hilbert polynomial of $X$ is $P_{X}(t)=2 r t+a-r$, and so its arithmetic genus $g_{X}$ is equal to $r+1-a$.

Proof. By construction, the curves $C$ and $X$ and the invertible sheaf $\mathcal{L}$ are related via the short exact sequence (2), and so the Hilbert polynomial $P_{X}(t)$ of $X$ is equal to the sum of the Hilbert polynomial $P_{C}(t)$ of $C$ and of the Euler characteristic $\chi \mathcal{L} \otimes \mathcal{O}_{\mathbb{P}^{n}}(t)$. By restriction to $\mathbb{P}^{1}$ we get $\chi \mathcal{L} \otimes \mathcal{O}_{\mathbb{P}^{n}}(t)=\chi \mathcal{O}_{\mathbb{P}^{1}}(r t-r-2+a)=r t-r-1+a$. The Hilbert polynomial of $C$ is equal to $P_{C}(t)=r t+1$, and so the claim follows.

Now, we describe a parameter space for the doublings of the rational normal curves of fixed degree and genus.

From Proposition 2.11, it follows that if we fix degree and genus of $X$ then we fix the degree $r$ of the rational normal curve $C=X_{\text {red }}$ and the twist $a=r+1-g \in \mathbb{Z}$ for the $\operatorname{map} \mu: \mathcal{O}_{\mathbb{P}^{1}}^{r-1}(-r-2) \oplus \mathcal{O}_{\mathbb{P}^{1}}^{n-r}(-r) \rightarrow \mathcal{O}_{\mathbb{P}^{1}}(-r-2+a)=\mathcal{O}_{\mathbb{P}^{1}}(-1-g)$.

Let $P(t)=2 r t+1-g$ be a polynomial and let $\mathcal{H} i l b_{p(t)}\left(\mathbb{P}^{n}\right)$ be the Hilbert scheme parameterizing locally Cohen-Macaulay curves of $\mathbb{P}^{n}$ with Hilbert polynomial $P(t)$. Let $H(2 r, g, n)$ be the locus in $\mathcal{H} i l b_{p(t)}\left(\mathbb{P}^{n}\right)$ whose closed points correspond to double structures 
of genus $g$ on smooth rational normal curves of degree $r$ embedded in $\mathbb{P}^{n}$. Let $H(r, n)$ be the locus in $\mathcal{H i l b}_{r t+1}\left(\mathbb{P}^{n}\right)$ whose closed points are smooth rational normal curves of degree $r$ in $\mathbb{P}^{n}$. $H(r, n)$ is open in an irreducible component of $\mathcal{H i l b}_{r t+1}\left(\mathbb{P}^{n}\right)$ of dimension $\operatorname{dim} P G L_{r}-\operatorname{dim} P G L_{1}+\operatorname{dim} \operatorname{Grass}(n-r, n)=(n+1)(r+1)-4$, where $\operatorname{Grass}(n-r, n)$ is the Grassmannian of the linear spaces of dimension $n-r$ in $\mathbb{P}^{n}$. Furthermore, there is a natural map $\varphi: H(2 r, g, n) \rightarrow H(r, n)$ defined as $\varphi(X)=X_{\text {red }}$ where, with abuse of notation, we denote $X$ both the subscheme in $\mathbb{P}^{n}$ and the closed point in the Hilbert scheme. The fibers of $\varphi$ are isomorphic to

$$
\operatorname{Hom}\left(\mathcal{O}_{\mathbb{P}^{1}}^{r-1}(-r-2) \oplus \mathcal{O}_{\mathbb{P}^{1}}^{n-r}(-r) \rightarrow \mathcal{O}_{\mathbb{P}^{1}}(-1-g)\right) / \operatorname{Aut}\left(\mathcal{O}_{\mathbb{P}^{1}}(-1-g)\right)
$$

and hence they are irreducible and smooth of dimension $(n-1)(r+1-g)+2 r-2-n$. Moreover, both $H(2 r, g, n)$ and $H(r, n)$ are stable under the action of $P G L_{n}$, and so we have proved the following

Theorem 2.12. $H(2 r, g, n)$ is irreducible of dimension

$$
\operatorname{dim} H(2 r, g, n)=(n+1)(2 r+1-g)-7+2 g .
$$

Remark 2.13. Of course, we do not know if $H(2 r, g, n)$ is an irreducible component of $\mathcal{H} i l b_{P(t)}\left(\mathbb{P}^{n}\right)$. It is reasonable that, under suitable hypotheses on $g$, it is so. In the last section of the paper, we will study the local properties of $H(4, g, n)$ for $n \geq 3$.

Corollary 2.14. Every irreducible component of $\mathcal{H} i l b_{2 r t+1-g}\left(\mathbb{P}^{n}\right)$ containing $H(2 r, g, n)$ has dimension $\geq(n+1)(2 r+1-g)-7+2 g$.

\section{Cohomology estimates}

In this section, we show how to compute the Rao function of a double rational normal curve in terms of the data of the construction. To achieve the results, we have to investigate also the curve $D$ defined by the ideal sheaf $\mathcal{I}_{C}^{2}$.

At first, we restate a deep result about $D$ due to J. Wahl (see [29], Theorem 2.1), that holds more generally for every Veronese embedding of a projective space.

Theorem 3.1. Let $C$ be a rational normal curve. Then, $H^{1} \mathcal{I}_{C}^{2}(j)=0$ for $j \neq 2$.

We want to compute $h^{1} \mathcal{I}_{C}^{2}(2)$ and the ideal $I_{D}=\left(I_{C}^{2}\right)^{\text {sat }}$. The following results are probably known in literature, but we add their proofs for completeness. The computation of the generators of $I_{D}$ rests on some direct calculations and on some basic results on initial ideals. Let us recall the following

Lemma 3.2. Let $I, J$ be ideals in a polynomial ring $R$. If $I \subseteq J$ and $\operatorname{in}(I) \supseteq \operatorname{in}(J)$, then $I=J$, no matter what term ordering we use to compute the initial ideal.

Proof. If $I \subseteq J$ then $\operatorname{in}(I) \subseteq \operatorname{in}(J)$. From our hypothesis, it follows that $\operatorname{in}(I)=\operatorname{in}(J)$, and the claim follows from ([9], Lemma 15.5).

Now, we prove some results about the initial ideal of the ideal of a rational normal curve and of its square. 
Lemma 3.3. Let $C \subset \mathbb{P}^{r}$ be the rational normal curve of degree $r$ generated by the $2 \times 2$ minors of the matrix

$$
A=\left(\begin{array}{cccc}
x_{0} & x_{1} & \ldots & x_{r-1} \\
x_{1} & x_{2} & \ldots & x_{r}
\end{array}\right)
$$

Then, with respect to the degrevlex ordering of the terms in $R$, we have that

(1) $\operatorname{in}\left(I_{C}\right)=\left\langle x_{1}, \ldots, x_{r-1}\right\rangle^{2}$;

(2) $\operatorname{in}\left(I_{C}^{2}\right)=\left\langle x_{1}, \ldots, x_{r-1}\right\rangle^{4}+x_{0}\left\langle x_{2}, \ldots, x_{r-1}\right\rangle^{3}+x_{r}\left\langle x_{2}, \ldots, x_{r-2}\right\rangle^{3}$;

(3) $\left(i n\left(I_{C}^{2}\right)\right)^{s a t}=i n\left(I_{C}^{2}\right)+\left\langle x_{2}, \ldots, x_{r-2}\right\rangle^{3}$.

Proof. Let $f_{i j}$ be the minor given by the $i$-th and $j$-th columns of $A$, i.e. $f_{i j}=x_{i-1} x_{j}-$ $x_{i} x_{j-1}$, with $1 \leq i<j \leq r$. The leading term of $f_{i j}$, with respect to the graded reverse lexicographic order, is $\operatorname{in}\left(f_{i j}\right)=x_{i} x_{j-1}$, and so $\operatorname{in}\left(I_{C}\right) \supseteq\left\langle x_{1}, \ldots, x_{r-1}\right\rangle^{2}$. On the other hand, it is easy to verify that $\left\{f_{12}, \ldots, f_{r-1, r}\right\}$ is a Gröbner basis of $I_{C}$ and so the first claim holds.

Let $D \subset \mathbb{P}^{r}$ be the curve defined by the ideal $I_{D}=H_{*}^{0}\left(\mathcal{I}_{C}^{2}\right)=\left(I_{C}^{2}\right)^{\text {sat }}$. Thanks to the previous Theorem, the homogeneous parts of the ideals $I_{C}$ and $I_{D}$ are related each other from the exact sequence

$$
0 \rightarrow\left(I_{D}\right)_{t} \rightarrow\left(I_{C}\right)_{t} \rightarrow H^{0}\left(\mathcal{O}_{\mathbb{P}^{1}}^{r-1}(-r-2+r t)\right) \rightarrow 0
$$

for every $t \geq 3$. Hence, $\operatorname{dim}_{K}\left(I_{D}\right)_{t}=\left(\begin{array}{c}r+t \\ r\end{array}\right)-\left(r^{2} t+2-r^{2}\right)$. It is well known that $\operatorname{dim}_{K}(I)_{t}=$ $\operatorname{dim}_{K}(\operatorname{in}(I))_{t}$ for every homogeneous ideal ([9], Theorem 15.26). So, $\operatorname{dim}_{K}\left(\operatorname{in}\left(I_{C}^{2}\right)\right)_{t} \leq$ $\left(\begin{array}{c}r+t \\ r\end{array}\right)-\left(r^{2} t+2-r^{2}\right)$.

Consider the monomial ideal $J=\left\langle x_{1}, \ldots, x_{r-1}\right\rangle^{4}+x_{0}\left\langle x_{2}, \ldots, x_{r-1}\right\rangle^{3}+x_{r}\left\langle x_{2}, \ldots, x_{r-2}\right\rangle^{3}$.

We claim that $J=\operatorname{in}\left(I_{C}^{2}\right)$.

It is a straightforward computation to check that

$$
\operatorname{dim}_{K}(J)_{t}=\left(\begin{array}{c}
r+t \\
r
\end{array}\right)-\left(r^{2} t+2-r^{2}\right),
$$

for $t \geq 4$. For example, it is easy to enumerate the degree $t$ monomials not in $J$. It follows that $\operatorname{dim}_{K}\left(\operatorname{in}\left(I_{C}^{2}\right)\right)_{t} \leq \operatorname{dim}_{K}(J)_{t}$. So, if $\operatorname{in}\left(I_{C}^{2}\right) \supseteq J$, then $\operatorname{in}\left(I_{C}^{2}\right)=J$. Furthermore, we get also that $\left(I_{D}\right)_{t}=\left(I_{C}^{2}\right)_{t}$ for $t \geq 4$, because their homogeneous parts have the same dimension for $t \geq 4$.

Of course, $\operatorname{in}\left(I_{C}^{2}\right) \supset\left\langle x_{1}, \ldots, x_{r-1}\right\rangle^{4}$, because of the first claim. Moreover, if $2 \leq i \leq$ $j \leq h \leq r-1$, the leading term of $f_{1 i} f_{j, h+1}-f_{1, j+1} f_{i-1, h+1}$ is equal to $x_{0} x_{i} x_{j} x_{h}$ and so $x_{0}\left\langle x_{2}, \ldots, x_{r-1}\right\rangle^{3} \subset \operatorname{in}\left(I_{C}^{2}\right)$. Finally, if $2 \leq i \leq j \leq h \leq r-2$, then the leading term of $f_{i, j+1} f_{h+1, r}-f_{i, h+2} f_{j, r}+f_{i-1, r} f_{j+1, h+2}$ is equal to $x_{i} x_{j} x_{h} x_{r}$ and so $x_{r}\left\langle x_{2}, \ldots, x_{r-2}\right\rangle^{3} \subset$ $\operatorname{in}\left(I_{C}^{2}\right)$. Because of the previous argument, the second claim follows. The equality in (3) follows from the definition of saturation.

Now, we can compute the generators of $I_{D}$.

Theorem 3.4. With the same hypotheses as before, let $I^{\prime}$ be the ideal generated by the $3 \times 3$ minors of the matrix

$$
B=\left(\begin{array}{cccc}
x_{0} & x_{1} & \ldots & x_{r-2} \\
x_{1} & x_{2} & \ldots & x_{r-1} \\
x_{2} & x_{3} & \ldots & x_{r}
\end{array}\right)
$$


Then, $I_{D}=I_{C}^{2}+I^{\prime}$.

Proof. By construction, in $\left(I^{\prime}\right) \supseteq\left\langle x_{2}, \ldots, x_{r-2}\right\rangle^{3}$, and by Lemma $3.3(3)$, we have $\left(\operatorname{in}\left(I_{C}^{2}\right)\right)^{\text {sat }}$ $=$ in $\left(I_{C}^{2}\right)+\left\langle x_{2}, \ldots, x_{r-2}\right\rangle^{3}$. Moreover, we have the following chain of inclusions $\operatorname{in}\left(I_{C}^{2}+I^{\prime}\right) \supseteq \operatorname{in}\left(I_{C}^{2}\right)+\operatorname{in}\left(I^{\prime}\right) \supseteq \operatorname{in}\left(I_{C}^{2}\right)+\left\langle x_{2}, \ldots, x_{r-2}\right\rangle^{3}=\left(\operatorname{in}\left(I_{C}^{2}\right)\right)^{s a t} \supseteq \operatorname{in}\left(\left(I_{C}^{2}\right)^{\text {sat }}\right)=\operatorname{in}\left(I_{D}\right)$.

So, from the inclusion $I_{C}^{2} \subseteq I_{D}$ and Lemma 3.2, it is enough to show that $I^{\prime} \subset I_{D}$.

Let us consider the integers $i, j, h$ with $2 \leq i<j<h \leq r$ and let

$$
g_{i j h}=\operatorname{det}\left(\begin{array}{ccc}
x_{i-2} & x_{j-2} & x_{h-2} \\
x_{i-1} & x_{j-1} & x_{h-1} \\
x_{i} & x_{j} & x_{h}
\end{array}\right) .
$$

It is evident that the two following equalities hold

$$
g_{i j h}=x_{i-2} f_{j h}-x_{j-2} f_{i h}+x_{h-2} f_{i j}=x_{i} f_{j-1, h-1}-x_{j} f_{i-1, h-1}+x_{h} f_{i-1, j-1} .
$$

We want to prove that $x_{k} g_{i j h} \in I_{C}^{2}$ for every $k=0, \ldots, r$.

From Proposition 2.1, we know that

$$
x_{i-1} f_{j h}-x_{j-1} f_{i h}+x_{k-1} f_{i j}=x_{i} f_{j h}-x_{j} f_{i h}+x_{h} f_{i j}=0 .
$$

Hence, the following easy computations prove the claim

$$
x_{k} g_{i j h}=\left\{\begin{array}{cl}
f_{i-1, k} f_{j h}-f_{j-1, k} f_{i h}+f_{h-1, k} f_{i j} & \text { if } k \geq h \\
f_{i, k+1} f_{j-1, k-1}-f_{j, k+1} f_{i-1, h-1}+f_{k+1, h} f_{i-1, j-1} & \text { if } k<h
\end{array}\right.
$$

where, in the last equation, we use the convention that $f_{a a}=0$ for every $a$, and $f_{a b}=f_{b a}$ if $a>b$.

Remark 3.5. If $C$ is a line or a smooth conic in $\mathbb{P}^{n}$ then $I_{C}^{2}$ is generated by $\left(\begin{array}{l}r \\ 2\end{array}\right)$ polynomials. By the way, those two cases are the only for which $I_{C}$ is a complete intersection ideal. If $C$ is a twisted cubic curve, then $I_{C}^{2}$ is saturated. If $r \geq 4$, then $I_{C}^{2}$ is no more saturated, but $I_{C}^{2}$ and its saturation agree from degree 4 on.

Remark 3.6. In the proof of Theorem 2.6, we checked that $\psi\left(j^{*}\left(\tilde{\varepsilon}_{2} \otimes i d_{\mathcal{O}_{C}}\right)\left(e_{i} \wedge e_{j} \wedge e_{h} \otimes\right.\right.$ $\left.\left.f_{k}\right)\right)=0$, and so $j^{*}\left(\tilde{\varepsilon}_{2} \otimes i d_{\mathcal{O}_{C}}\right)\left(e_{i} \wedge e_{j} \wedge e_{h} \otimes f_{k}\right) \in \operatorname{ker}\left(\mu_{\mid} \circ \psi\right)$ where $\mu_{\mid}$is the restriction of $\mu$ to $\wedge^{2} \mathcal{O}_{\mathbb{P} 1}^{r-1}(-r-2)$. The element $j^{*}\left(\tilde{\varepsilon}_{2} \otimes i d_{\mathcal{O}_{C}}\right)\left(e_{i} \wedge e_{j} \wedge e_{h} \otimes f_{k}\right)$ corresponds to the minor $g_{i j h}$ and so $\left(I_{C}^{2}\right)^{\text {sat }} \subseteq I_{X}$ for every double structure $X$.

Now, we consider the case $C \subset \mathbb{P}^{r} \subset \mathbb{P}^{n}$. As in section 2, we denote $S=K\left[x_{0}, \ldots, x_{r}\right]$, and $R=K\left[x_{0}, \ldots, x_{n}\right]$.

Proposition 3.7. Let $C \subset L \cong \mathbb{P}^{r} \subset \mathbb{P}^{n}$ be a rational normal curve. Let $I_{C, L}, I_{C}$ be the ideals of $C$ as a subscheme of $L$ and of $\mathbb{P}^{n}$, respectively, and let $I_{L}$ be the ideal of $L$ in $\mathbb{P}^{n}$. Then, $\left(I_{C}^{2}\right)^{\text {sat }}=\left(\left(I_{C, L}^{2}\right)^{\text {sat }}\right)^{e}+\left(I_{C, L}^{e}\right) \cdot I_{L}+I_{L}^{2}=\left(\left(I_{C, L}^{2}\right)^{\text {sat }}\right)^{e}+I_{C} \cdot I_{L}$ where the extension is via the natural inclusion $S \hookrightarrow R$.

Proof. The ideals $I_{L}^{2}$ and $\left(I_{C, L}^{e}\right) \cdot I_{L}$ are obviously contained in $I_{C}^{2}$ and hence in $\left(I_{C}^{2}\right)^{s a t}$. Furthermore, to check the inclusion $\left(\left(I_{C, L}^{2}\right)^{s a t}\right)^{e} \subset\left(I_{C}^{2}\right)^{\text {sat }}$ it is enough to verify that $f \in$ $\left(I_{C}^{2}\right)^{s a t}$ for every $f \in\left(\left(I_{C, L}^{2}\right)^{s a t}\right)^{e} \cap S$. Let $f$ be a homogeneous polynomial in $\left(\left(I_{C, L}^{2}\right)^{s a t}\right)^{e} \cap S$. It is easy to check that, for every $i=0, \ldots, n$, there exists $m_{i} \in \mathbb{N}$ such that $x_{i}^{m_{i}} f \in\left(I_{C}^{2}\right)$. In fact, if $0 \leq i \leq r$, then there exists $m_{i} \in \mathbb{N}$ such that $x_{i}^{m_{i}} f \in\left(I_{C, L}^{2}\right)^{e} \cap S \subseteq\left(I_{C}^{2}\right)$, 
while, for $r+1 \leq i \leq n, x_{i}^{2} f \in I_{C}^{2}$ because $I_{L}^{2} \subset I_{C}^{2}$. Hence, $\left(\left(I_{C, L}^{2}\right)^{\text {sat }}\right)^{e} \subseteq\left(I_{C}^{2}\right)^{\text {sat }}$ and the inclusion $\left(\left(I_{C, L}^{2}\right)^{\text {sat }}\right)^{e}+\left(I_{C, L}^{e}\right) \cdot I_{L}+I_{L}^{2} \subseteq\left(I_{C}^{2}\right)^{\text {sat }}$ follows.

To prove the inverse inclusion, let $f \in\left(I_{C}^{2}\right)^{\text {sat }}$ be a homogeneous polynomial. There exist $f_{1} \in S$ and $f_{2} \in I_{L}$ such that $f=f_{1}+f_{2}$ and the decomposition is unique. For every $i=0, \ldots, n$, there exists $m_{i} \in \mathbb{N}$ such that $x_{i}^{m_{i}} f \in I_{C}^{2}$. Assume $0 \leq i \leq r$. We know that $I_{C}=I_{C, L}^{e}+I_{L}$, and so $I_{C}^{2}=\left(I_{C, L}^{2}\right)^{e}+I_{L} \cdot I_{C}$. Hence, there exist $g_{1} \in\left(I_{C, L}^{2}\right)^{e} \cap S$ and $g_{2} \in I_{L} \cdot I_{C}$ such that $x_{i}^{m_{i}} f=x_{i}^{m_{i}} f_{1}+x_{i}^{m_{i}} f_{2}=g_{1}+g_{2}$. It is evident that $x_{i}^{m_{i}} f_{1}-g_{1}=$ $g_{2}-x_{i}^{m_{i}} f_{2} \in S \cap I_{L}=0$, and so $x_{i}^{m_{i}} f_{1} \in\left(I_{C, L}^{2}\right)^{e}$ and $x_{i}^{m_{i}} f_{2} \in I_{L} \cdot I_{C}$. By definition of saturation, $f_{1} \in\left(\left(I_{C, L}^{2}\right)^{e}\right)^{s a t}$. By assumption, $f_{2} \in I_{L}$, and so $f_{2}=x_{r+1} f_{2, r+1}+\cdots+x_{n} f_{2, n}$. Then, $x_{i}^{m_{i}} f_{2, j} \in I_{C}$ for every $j=r+1, \ldots, n$. The ideal $I_{C}$ is a prime ideal and $x_{i}^{m_{i}} \notin I_{C}$. Hence, $f_{2, j} \in I_{C}, f_{2} \in I_{L} \cdot I_{C}$ and the proof is complete.

A consequence of the previous results is that we can compute also $h^{1} \mathcal{I}_{C}^{2}(2)$.

Corollary 3.8. With the same hypotheses as before, $h^{1} \mathcal{I}_{C}^{2}(2)=\left(\begin{array}{c}r-1 \\ 2\end{array}\right)$.

Proof. If we tensor the exact sequence

$$
0 \rightarrow \mathcal{I}_{C}^{2} \rightarrow \mathcal{I}_{C} \rightarrow \mathcal{O}_{\mathbb{P}^{1}}^{r-1}(-r-2) \oplus \mathcal{O}_{\mathbb{P}^{1}}^{n-r}(-r) \rightarrow 0
$$

by $\mathcal{O}_{\mathbb{P}^{n}}(2)$ and we take the cohomology, we get

$$
h^{1} \mathcal{I}_{C}^{2}(2)=h^{0} \mathcal{I}_{C}^{2}(2)-h^{0} \mathcal{I}_{C}(2)+(r-1) h^{0} \mathcal{O}_{\mathbb{P}^{1}}(r-2)+(n-r) h^{0} \mathcal{O}_{\mathbb{P}^{1}}(r)=\left(\begin{array}{c}
r-1 \\
2
\end{array}\right) .
$$

Thanks to the results on $D$, we can compute, or at least bound, the Rao function $h^{1} \mathcal{I}_{X}(j), j \in \mathbb{Z}$, of $X$ in terms of the map $\mu$ which describes the schematic structure of $X$.

Proposition 3.9. With the same notation as above, let $I_{\mu}$ be the ideal generated by the entries of $\mu$. Then, it holds

$$
h^{1} \mathcal{I}_{X}(j)=\operatorname{dim}_{K}\left(\frac{K[t, u]}{I_{\mu}}\right)_{r j-r-2+a}
$$

for every $j \neq 2$. Moreover,

$$
h^{1} \mathcal{I}_{X}(2) \leq \operatorname{dim}_{K}\left(\frac{K[t, u]}{I_{\mu}}\right)_{r-2+a}+\left(\begin{array}{c}
r-1 \\
2
\end{array}\right) .
$$

Proof. By construction, we have the short exact sequences

$$
0 \rightarrow \mathcal{I}_{X} \rightarrow \mathcal{I}_{C} \rightarrow \mathcal{L} \rightarrow 0
$$

and

$$
0 \rightarrow \frac{\mathcal{I}_{X}}{\mathcal{I}_{C}^{2}} \rightarrow \frac{\mathcal{I}_{C}}{\mathcal{I}_{C}^{2}} \rightarrow \mathcal{L} \rightarrow 0
$$

where the first map of them both is the inclusion. 
The two sequences fit into the larger commutative diagram

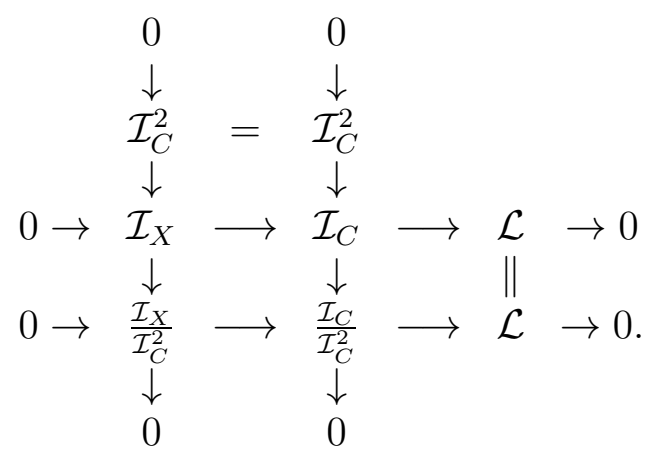

If we twist by $\mathcal{O}_{\mathbb{P}^{n}}(j)$ and take the cohomology, we get

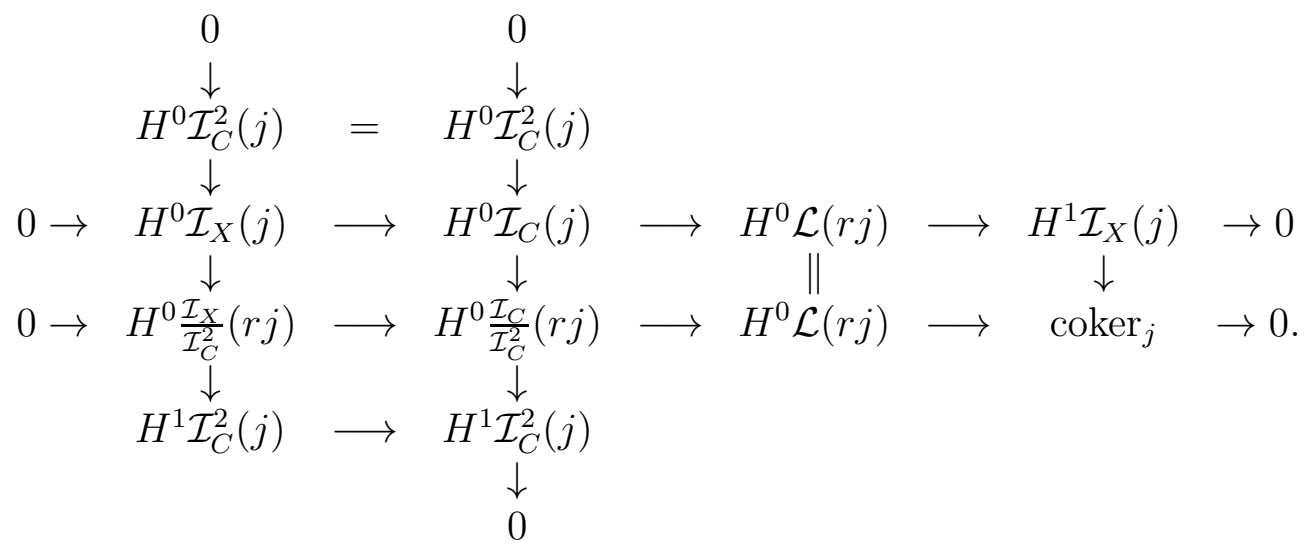

If $j \neq 2$, then $H^{1} \mathcal{I}_{C}^{2}(j)=0$ and so

$$
\operatorname{coker}\left(H^{0} \mathcal{I}_{X}(j) \rightarrow H^{0} \mathcal{I}_{C}(j)\right)=\operatorname{coker}\left(H^{0} \frac{\mathcal{I}_{X}}{\mathcal{I}_{C}^{2}}(r j) \rightarrow H^{0} \frac{\mathcal{I}_{C}}{\mathcal{I}_{C}^{2}}(r j)\right)
$$

as subspaces of $H^{0} \mathcal{L}(r j)$. Hence, $H^{1} \mathcal{I}_{X}(j) \cong \operatorname{coker}_{j} \cong\left(\frac{K[t, u]}{I_{\mu}}\right)_{r j-r-2+a}$.

If $j=2$, we set $\mathcal{A}=\operatorname{ker}\left(H^{0} \mathcal{L}(2 r) \rightarrow H^{1} \mathcal{I}_{X}(2)\right)$ and $\mathcal{B}=\operatorname{ker}\left(H^{0} \mathcal{L}(2 r) \rightarrow\right.$ coker $\left._{2}\right)$. Then, the identity of $H^{0} \mathcal{L}(2 r)$ induces an injective map $\mathcal{A} \rightarrow \mathcal{B}$, and the diagram

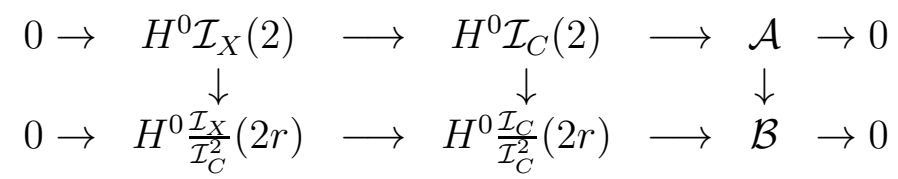

induces a surjective map

$$
H^{1} \mathcal{I}_{C}^{2}(2) \rightarrow \operatorname{coker}(\mathcal{A} \rightarrow \mathcal{B}) \cong \operatorname{ker}\left(H^{1} \mathcal{I}_{X}(2) \rightarrow \operatorname{coker}_{2}\right) .
$$

Hence, the claim follows from the surjectivity of the map $H^{1} \mathcal{I}_{X}(2) \rightarrow$ coker $_{2}$.

Example 3.10 (Example 2.9] revisited). The genus of the curve $X$ is $g_{X}=4-1=3$, because $a=1$ (see Proposition 2.11). The map $\mu: \mathcal{O}_{\mathbb{P}^{1}}^{2}(-5) \rightarrow \mathcal{O}_{\mathbb{P}^{1}}(-4)$ was defined as $\mu=(t, u)$ and so the Hilbert function of its cokernel is

$$
\operatorname{dim}_{K}\left(\frac{K[t, u]}{(t, u)}\right)_{h}= \begin{cases}1 & \text { if } h=0 \\ 0 & \text { otherwise }\end{cases}
$$


By Proposition 3.9, the Rao function of $X$ is equal to

$$
h^{1} \mathcal{I}_{X}(j)=\operatorname{dim}_{K}\left(\frac{K[t, u]}{(t, u)}\right)_{3 j-4}=0
$$

for every $j \neq 2$. To compute $h^{1} \mathcal{I}_{X}(2)$ we consider the hyperplane $H=V(w)$ that is general for $X$, and the exact sequence

$$
0 \rightarrow \mathcal{I}_{X}(-1) \rightarrow \mathcal{I}_{X} \rightarrow \mathcal{I}_{X \cap H \mid H} \rightarrow 0 .
$$

If we tensorize by $\mathcal{O}_{\mathbb{P}^{3}}(2)$ and take the cohomology, we get

$$
0 \rightarrow H^{1} \mathcal{I}_{X}(2) \rightarrow H^{1} \mathcal{I}_{X \cap H \mid H}(2) \rightarrow 0,
$$

because $H^{2} \mathcal{I}_{X}(1)=0$. It is easy to verify that $h^{1} \mathcal{I}_{X \cap H \mid H}(2)=h^{0} \mathcal{I}_{X \cap H \mid H}(2)$ and so $h^{1} \mathcal{I}_{X}(2)=0$ if, and only if, $X \cap H$ is not contained in any conic of $H$. But $\left(\frac{I_{X}+w R}{w R}\right)^{s a t}=$ $\left\langle 2 y^{3}-3 x y z, y^{2} z-2 x z^{2}, y z^{2}, z^{3}\right\rangle$ and so $X$ is an arithmetically Cohen-Macaulay curve in $\mathbb{P}^{3}$.

\section{Arithmetically Gorenstein double Rational normal Curves}

In this section, we want to describe the arithmetically Gorenstein curves among the double structures on rational normal curves. At first, we characterize the possible triples $(2 r, g, n)$ and then we study the possible cases one at a time.

To start, we recall the definition of arithmetically Gorenstein curve.

Definition 4.1. A curve $X \subset \mathbb{P}^{n}$ is arithmetically Gorenstein if its homogeneous coordinate ring $R_{X}$ is a Gorenstein ring, or, equivalently, if $R_{X}$ is Cohen-Macaulay and its canonical sheaf $\omega_{X}$ is a twist of the structure sheaf.

Now, we look for triples $(2 r, g, n)$ for which the property of being arithmetically Gorenstein is allowed.

Proposition 4.2. Let $C \subset L \cong \mathbb{P}^{r} \subset \mathbb{P}^{n}, n \geq 3$, be a rational normal curve of degree $r$, let $\mu: \mathcal{O}_{\mathbb{P}^{1}}^{r-1}(-r-2) \oplus \mathcal{O}_{\mathbb{P}^{1}}^{n-r}(-r) \rightarrow \mathcal{O}_{\mathbb{P}^{1}}(-1-g)$ be a surjective map, and let $X$ be the double structure on $C$ defined by $\mu$. If $X$ is a non-degenerate arithmetically Gorenstein curve, then either $(2 r, g, n)=(2 r, r+1, r)$ or $(2 r, g, n)=(2 r, 1,2 r-1)$.

Proof. If $X$ is an arithmetically Gorenstein curve, then the second difference $\Delta^{2} h_{X}$ of its Hilbert function $h_{X}$ is a symmetric function. Moreover, if $X$ is non-degenerate, then $\Delta^{2} h_{X}(1)=n-1$. We have the equality $2 r=\operatorname{deg}(X)=\sum_{j=0}^{\infty} \Delta^{2} h_{X}(j)$ and so we get $r \leq n \leq 2 r$, where the first inequality comes from the general setting, and the second one from $\Delta^{2} h_{X}(0)=1$. If $X$ is an arithmetically Gorenstein curve, then $\Delta^{2} h_{X}$ is the Hilbert function of the Artinian ring $R /\left\langle I_{X}, h_{1}, h_{2}\right\rangle$ where $h_{1}, h_{2}$ are two linear forms, general with respect to $X$. In particular, if $\Delta^{2} h_{X}(j)=0$ for some $j>0$, then $\Delta^{2} h_{X}(k)=0$ for every $k \geq$ $j$. From the above discussion and inequalities, we get that there are either 3 or 4 non-zero entries in $\Delta^{2} h_{X}$. In the first case, then $\Delta^{2} h_{X}=(1,2 r-2,1)$ and $(2 r, g, n)=(2 r, 1,2 r-1)$. In the second case, then $\Delta^{2} h_{X}=(1, r-1, r-1,1)$ and $(2 r, g, n)=(2 r, r+1, r)$.

Remark 4.3. If $X$ is a double conic in $\mathbb{P}^{2}$, then $I_{X}=\left\langle q^{2}\right\rangle$ where $q=x z-y^{2}$ defines the smooth conic that supports $X . X$ is arithmetically Gorenstein with $\omega_{X}=\mathcal{O}_{X}(1)$. 
Now, we characterize the arithmetically Gorenstein double curves among the ones we can construct with given triple $(2 r, g, n)$.

Theorem 4.4. Let $(2 r, g, n)=(2 r, r+1, r)$. For every non-zero map $\mu: \mathcal{O}_{\mathbb{P} 1}^{r-1}(-r-2) \rightarrow$ $\mathcal{O}_{\mathbb{P}^{1}}(-r-2)$ we get a non-degenerate arithmetically Cohen-Macaulay curve X. Furthermore, if $\mu$ is general, then $X$ is arithmetically Gorenstein. If $\mu=\left(\alpha^{r-2}, \alpha^{r-3} \beta, \ldots, \beta^{r-2}\right)$ then $X$ is contained in a cone over a rational normal curve $C^{\prime} \subset \mathbb{P}^{r-1}$.

Proof. Let $\mu=\left(a_{0}, \ldots, a_{r-2}\right) \neq 0$ with $a_{i} \in K$. Let $X$ be the curve we get by doubling a rational normal curve $C$ via $\mu$.

We prove that $X$ is an arithmetically Cohen-Macaulay curve. By Proposition [3.9, the surjectivity of $\mu$ implies that $h^{1} \mathcal{I}_{X}(j)=0$ for $j \neq 2$, and so we have to prove that $h^{1} \mathcal{I}_{X}(2)=0$, too. The map $\mu \circ \psi$ can be written also as the composition $\mu^{\prime} \circ \psi^{\prime}$ where $\mu^{\prime}$ : $\mathcal{O}_{\mathbb{P} 1}^{r-1}(-2 r) \rightarrow \mathcal{O}_{\mathbb{P}^{1}}(-r-2)$ is defined as $\mu^{\prime}=\left(t^{r-2}, t^{r-3} u, \ldots, u^{r-2}\right)$ and $\psi^{\prime}: \wedge^{2} \mathcal{O}_{\mathbb{P} 1}^{r}(-r) \rightarrow$ $\mathcal{O}_{\mathbb{P}^{1}}^{r-1}(-2 r)$ is defined by the matrix obtained from the one of $\psi$ by substituting $t^{i} u^{j}, i+j=$ $r-2$, with $a_{j}$, where $\psi$ was defined in Theorem 2.6. The matrix of $\psi^{\prime}$ is full rank for whatever non-zero map $\mu$, and so it has exactly $\left(\begin{array}{l}r \\ 2\end{array}\right)-(r-1)=\left(\begin{array}{c}r-1 \\ 2\end{array}\right)$ linearly independent degree 0 syzygies. Furthermore, a degree 0 syzygy of $\mu \circ \psi$ is a degree 0 syzygy of $\psi^{\prime}$ and hence, $h^{0} \mathcal{I}_{X}(2)=\left(\begin{array}{c}r-1 \\ 2\end{array}\right)$. From the exact sequence

$$
0 \rightarrow H^{0} \mathcal{I}_{X}(2) \rightarrow H^{0} \mathcal{I}_{C}(2) \rightarrow H^{0} \frac{\mathcal{I}_{C}}{\mathcal{I}_{X}}(2) \rightarrow H^{1} \mathcal{I}_{X}(2) \rightarrow 0,
$$

and from $h^{0} \mathcal{I}_{C}(2)=\left(\begin{array}{l}r \\ 2\end{array}\right), h^{0} \frac{\mathcal{I}_{C}}{\mathcal{I}_{X}}(2)=h^{0} \mathcal{O}_{\mathbb{P}^{1}}(r-2)=r-1, h^{0} \mathcal{I}_{X}(2)=\left(\begin{array}{c}r-1 \\ 2\end{array}\right)$ we get that $h^{1} \mathcal{I}_{X}(2)=0$.

The curve $C$ is rational normal and so $\operatorname{Pic}(C)=\mathbb{Z}$. We know that the line bundle $\mathcal{L}$ verifies $j^{*}(\mathcal{L})=\mathcal{O}_{\mathbb{P}^{1}}(-r-2)$ and so $\mathcal{L}=\omega_{C}(-1)$ where $\omega_{C}$ is the canonical sheaf of $C$. Hence, the curve $X$ is defined via the exact sequence

$$
0 \rightarrow \mathcal{I}_{X} \rightarrow \mathcal{I}_{C} \rightarrow \omega_{C}(-1) \rightarrow 0 .
$$

From the exact sequence, we can compute the Hilbert function of $X$ and its second difference. In particular, we get $\Delta^{2} h_{X}=(1, r-1, r-1,1)$, as expected.

Assume now that $\mu=\left(\alpha^{r-2}, \alpha^{r-3} \beta, \ldots, \beta^{r-2}\right)$ for some $(\alpha, \beta) \in K^{2} \backslash\{(0,0)\}$. The $2 \times 2$ minors of the matrix

$$
\left(\begin{array}{cccc}
\beta x_{0}-\alpha x_{1} & \beta x_{1}-\alpha x_{2} & \ldots & \beta x_{r-2}-\alpha x_{r-1} \\
\beta x_{1}-\alpha x_{2} & \beta x_{2}-\alpha x_{3} & \cdots & \beta x_{r-1}-\alpha x_{r}
\end{array}\right)
$$

define a cone in $\mathbb{P}^{r}$ over a rational normal curve of $\mathbb{P}^{r-1}$. We want to prove that they belong to $I_{X}$. To this end, let $1 \leq i<j \leq r-1$. The minor $F_{i, j}$ given by the $i$-th and $j$-th columns is equal to

$$
F_{i, j}=\operatorname{det}\left(\begin{array}{cc}
\beta x_{i-1}-\alpha x_{i} & \beta x_{j-1}-\alpha x_{j} \\
\beta x_{i}-\alpha x_{i+1} & \beta x_{j}-\alpha x_{j+1}
\end{array}\right)=\beta^{2} f_{i j}-\alpha \beta f_{i, j+1}-\alpha \beta f_{i+1, j}+\alpha^{2} f_{i+1, j+1}
$$

where $f_{p q}=x_{p-1} x_{q}-x_{p} x_{q-1}$ is a generator of $I_{C}$. The claim follows if we prove that

$$
\beta^{2} e_{i} \wedge e_{j}-\alpha \beta e_{i} \wedge e_{j+1}-\alpha \beta e_{i+1} \wedge e_{j}+\alpha^{2} e_{i+1} \wedge e_{j+1}
$$


is a syzygy of $\mu \circ \psi$ for every $1 \leq i<j \leq r-2$. Since $\mu\left(g_{h}\right)=\alpha^{r-h-1} \beta^{h-1}, h=1, \ldots, r-1$, where $g_{1}, \ldots, g_{r-1}$ is the canonical basis of $\mathcal{O}_{\mathbb{P}^{1}}^{r-1}(-r-2)$, then, we have

$$
\begin{aligned}
\mu \circ \psi & \left(\beta^{2} e_{i} \wedge e_{j}-\alpha \beta e_{i} \wedge e_{j+1}-\alpha \beta e_{i+1} \wedge e_{j}+\alpha^{2} e_{i+1} \wedge e_{j+1}\right)= \\
=\mu & \left(t^{r-j-1} u^{j-1}\left(-\alpha \beta g_{i}+\alpha^{2} g_{i+1}\right)+t^{r-j} u^{j-2}\left(\beta^{2} g_{i}-2 \alpha \beta g_{i+1}+\alpha^{2} g_{i+2}\right)+\cdots+\right. \\
& \left.+t^{r-i-2} u^{i}\left(\beta^{2} g_{j-2}-2 \alpha \beta g_{j-1}+\alpha^{2} g_{j}\right)+t^{r-i-1} u^{i-1}\left(\beta^{2} g_{j-1}-\alpha \beta g_{j}\right)\right)=0
\end{aligned}
$$

and the claim follows.

Let $(\alpha, \beta)=(0,1)$. Then, as explained before, the double structure $X$ associated to $\mu=(0, \ldots, 0,1)$ is contained in the cone over the rational normal curve $C^{\prime}$ of $\mathbb{P}^{r-1} \cong H=$ $V\left(x_{r}\right)$ defined by the $2 \times 2$ minors of the matrix

$$
\left(\begin{array}{llll}
x_{0} & x_{1} & \ldots & x_{r-2} \\
x_{1} & x_{2} & \ldots & x_{r-1}
\end{array}\right) .
$$

The hyperplane $H$ intersects $C$ in $A(1: 0: \cdots: 0)$ with multiplicity $r$, and so $X \cap H$ is supported on $A$ and has degree $2 r$. Moreover, $X \cap H$ is contained in the rational normal curve $C^{\prime}$ and so it is arithmetically Gorenstein. In fact, $\operatorname{deg}(X \cap H)=2 \operatorname{deg}\left(C^{\prime}\right)+$ 2, $\operatorname{Pic}\left(C^{\prime}\right) \cong \mathbb{Z}$, and so $X \cap H \in\left|2 H^{\prime}-K^{\prime}\right|$, where $H^{\prime}$ is the class of a hyperplane section of $C^{\prime}$ and $K^{\prime}$ is the canonical divisor of $C^{\prime}$, and every divisor of the system $d H^{\prime}-K^{\prime}$ is arithmetically Gorenstein, for every $d \geq 0$ ([23], Theorem 4.2.8). Hence, $X$ is arithmetically Cohen-Macaulay with an arithmetically Gorenstein hyperplane section, i.e. $X$ is arithmetically Gorenstein, because the graded Betti numbers of the minimal free resolutions of $I_{X}$ and $I_{X \cap H \mid H}$ are the same. In fact, the only irreducible component of $X$ is $C$ that is non-degenerate, and so $x_{r}$ is not a 0 -divisor for $R / I_{X}$. Hence, the hyperplane $H=V\left(x_{r}\right)$ we considered is general enough for $X$ to let the proof of ([23], Theorem 1.3.6) work (see also [23], Remark 1.3.9).

The family $H(2 r, r+1, r)$ is irreducible, and the arithmetically Gorenstein locus in it is not empty. From the semicontinuity of the Betti numbers in an irreducible family ([7]) it follows that an open subscheme of $H(2 r, r+1, r)$ parametrizes arithmetically Gorenstein schemes and the claim follows.

Remark 4.5. In the case we just studied, the degree and the genus of $X$ are the ones of a canonical curve in $\mathbb{P}^{r}$, and so the result we proved is not unexpected. In fact, in [12], the author proved that $H(2 r, r+1, r)$ is contained in the closure of the component of the canonical curves.

Now, we consider the second case, namely $(2 r, g, n)=(2 r, 1,2 r-1)$.

Theorem 4.6. Let $(2 r, g, n)=(2 r, 1,2 r-1)$. Then for a general map $\mu: \mathcal{O}_{\mathbb{P} 1}^{r-1}(-r-2) \oplus$ $\mathcal{O}_{\mathbb{P} 1}^{r-1}(-r) \rightarrow \mathcal{O}_{\mathbb{P}^{1}}(-2)$ we get a non-degenerate arithmetically Gorenstein curve, where general means that $\mu_{\mid}: \mathcal{O}_{\mathbb{P}^{1}}^{r-1}(-r) \rightarrow \mathcal{O}_{\mathbb{P}^{1}}(-2)$ has no degree 0 syzygy.

Proof. Assume that the restriction $\mu_{1}$ of $\mu$ to $\mathcal{O}_{\mathbb{P} 1}^{r-1}(-r-2)$ is the null map.

If $r=2$, then $\mu=(0,1)$ and hence $X$ is defined by the ideal $I_{X}=\left(x_{0} x_{2}-x_{1}^{2}, x_{3}^{2}\right)$. Then $X$ is a complete intersection of a cone and a double plane.

Assume now that $r \geq 3$, and furthermore assume that the restriction $\mu_{2}$ of $\mu$ to $\mathcal{O}_{\mathbb{P}^{1}}^{r-1}(-r)$ is given by $\mu_{2}=\left(t^{r-2}, t^{r-3} u, \ldots, u^{r-2}\right)$. Of course, $\mu_{2}$ has no degree 0 syzygy. 
The map $\mu \circ \psi$ is given by the matrix $\left(0, \ldots, 0, t^{r-2}, t^{r-3} u, \ldots, u^{r-2}\right)$. By using the procedure described in section 2, we get that the double structure $X$ is defined by the ideal $I_{X}=I_{C, L}+I_{L}^{2}+J$ where $J=\left\langle x_{1} x_{r+1}-x_{0} x_{r+2}, \ldots, x_{r} x_{r+1}-x_{r-1} x_{r+2}, \ldots, x_{1} x_{2 r-2}-\right.$ $\left.x_{0} x_{2 r-1}, \ldots, x_{r} x_{2 r-2}-x_{r-1} x_{2 r-1}\right\rangle$. It is evident that the ideal $I_{S}$ defined by the $2 \times 2$ minors of the matrix

$$
\left(\begin{array}{cccccc}
x_{0} & \ldots & x_{r-1} & x_{r+1} & \ldots & x_{2 r-2} \\
x_{1} & \ldots & x_{r} & x_{r+2} & \ldots & x_{2 r-1}
\end{array}\right)
$$

is contained in $I_{X}$, i.e. $X$ is contained in $S$ which is a smooth rational normal scroll surface $\mathbb{P}\left(\mathcal{O}_{\mathbb{P}^{1}} \oplus \mathcal{O}_{\mathbb{P}^{1}}(-2)\right)$ embedded via the complete linear system $|\xi+r f|([9]$, exercise A2.22), where $\xi$ is the class of the rational normal curve of minimal degree $r-2$ contained in $S$ and $f$ is a fibre. On $S$, we have that $\xi^{2}=-2, \xi \cdot f=1, f^{2}=0$. Moreover, the canonical divisor of $S$ is $K_{S}=-2 \xi-2 f$, and the hyperplane section class is $H=\xi+r f$ ([17], Lemma 2.10). Then $X \in|a \xi+b f|$, with $a=2, b=4$, by adjunction, and so $X$ is an anticanonical divisor on $S$ and so it is a non-degenerate arithmetically Gorenstein curve ([23], Theorem 4.2.8). To complete the proof, we show that the curve $X$ we constructed before is the only double structure on $C$ of arithmetic genus 1, up to automorphisms of $\mathbb{P}^{2 r-1}$, which is the content of next Theorem 4.7.

Theorem 4.7. Let $C \subset L \cong \mathbb{P}^{r} \subset \mathbb{P}^{2 r-1}$ be a rational normal curve of degree $r$. Then, there exists only one non-degenerate double structure $X$ on $C$ of arithmetic genus 1 , up to automorphisms of $\mathbb{P}^{2 r-1}$.

Proof. To make the proof more readable, we choose the coordinates of $\mathbb{P}^{2 r-1}$ as $x_{0}, \ldots, x_{r}$, $y_{1}, \ldots, y_{r-1}$, where $L=V\left(y_{1}, \ldots, y_{r-1}\right)$.

As in the proof of the previous Theorem, let $\mu_{1}$ and $\mu_{2}$ be the restrictions of $\mu$ to $\mathcal{O}_{\mathbb{P} 1}^{r-1}(-r-2)$ and to $\mathcal{O}_{\mathbb{P} 1}^{r-1}(-r)$, respectively. Assume first that $\mu_{2}=\left(l_{1}, \ldots, l_{r-1}\right)$. The forms $l_{1}, \ldots, l_{r-1}$ are linearly dependent if, and only if, they have a degree 0 syzygy, that, of course, is also a degree 0 syzygy of $\mu \circ \psi$. So, $X$ is degenerate if, and only if, $l_{1}, \ldots, l_{r-1}$ are linearly dependent. Hence, we can assume that $l_{1}, \ldots, l_{r-1}$ are linearly independent, and so there exists an invertible matrix $P \in G L_{r-1}(K)$ such that

$$
\left(\begin{array}{c}
l_{1} \\
\vdots \\
l_{r-1}
\end{array}\right)=P\left(\begin{array}{c}
t^{r-2} \\
\vdots \\
u^{r-2}
\end{array}\right) .
$$

Going back to the construction, it is clear that the choice of the generators of $L$ plays no role when we restrict the maps to $\mathbb{P}^{1}$ and so, if we say that $I_{L}$ is generated by

$$
P^{-1}\left(\begin{array}{c}
y_{1} \\
\vdots \\
y_{r-1}
\end{array}\right)
$$

and we change bases in $\mathcal{O}_{\mathbb{P} 1}^{r-1}(-r)$ by using $P^{-1}$ we get that $I_{L}$ is generated by $y_{1}, \ldots, y_{r-1}$ and $\mu_{2}=\left(t^{r-2}, \ldots, u^{r-2}\right)$.

Let $\mu_{1}=\left(p_{1}, \ldots, p_{r-1}\right)$, where $p_{i}=p_{i 0} t^{r}+p_{i 1} t^{r-1} u+\cdots+p_{i r} u^{r}$. The map $\mu \circ \psi$ has the first $\left(\begin{array}{l}r \\ 2\end{array}\right)$ entries that are combinations of $t^{r-2}, \ldots, u^{r-2}$ with coefficients $p_{1}, \ldots, p_{r-1}$ and the last $r-1$ entries which are equal to $t^{r-2}, \ldots u^{r-2}$. Hence, the syzygies of $\mu \circ \psi$ 
can be easily computed and we get that the defining ideal $I_{X}$ of $X$ is generated by $x_{i-1} x_{i+1}-x_{i}^{2}-p_{i} y_{i}, i=1, \ldots, r-1$, by $x_{i} x_{j+1}-x_{i+1} x_{j}-y_{i+1} p_{j}-\cdots-y_{j} p_{i+1}, 0 \leq i<$ $j-1 \leq r-2$, by $x_{i} y_{j}-x_{i-1} y_{j+1}, i=1, \ldots, r, j=1, \ldots, r-2$, and by $y_{i} y_{j}, 1 \leq i \leq j \leq r-1$, where, with abuse of notation, we set $p_{i}$ also the only linear form in $x_{0}, \ldots, x_{r}$ that is equal to $p_{i}$ when restricted to $\mathbb{P}^{1}$, i.e. $p_{i}=p_{i 0} x_{0}+\cdots+p_{i r} x_{r}$.

We look for the required change of coordinates in the form

$$
x_{i}=z_{i}+a_{i 1} y_{1}+\ldots a_{i, r-1} y_{r-1} \quad i=0, \ldots, r
$$

and we fix the remaining variables $y_{1}, \ldots, y_{r-1}$.

Our goal is to prove that we can choose the $a_{i j}$ 's in such a way that, in the new coordinate system, $X$ is defined by the ideal $J$ generated by $z_{i-1} z_{i+1}-z_{i}^{2}, z_{i} z_{j+1}-z_{i+1} z_{j}, z_{i} y_{j}-$ $z_{i-1} y_{j+1}, y_{i} y_{j}$, where the indices vary in the same ranges as before.

If we apply the change of coordinates to the last generators of $I_{X}$ then they do not change, because the variables $y_{i}, \ldots y_{r-1}$ are fixed. If we apply the change of coordinates to the generators of the form $x_{i} y_{j}-x_{i-1} y_{j+1}$, we get that $z_{i} y_{j}-z_{i-1} y_{j+1} \in J$ because $y_{h} y_{k} \in J$, for $i=1, \ldots, r, j=1, \ldots, r-2$, and $1 \leq h \leq k \leq r-1$.

By applying the change of coordinates to $x_{i-1} x_{i+1}-x_{i}^{2}-y_{i} p_{i}$ we get

$$
\begin{aligned}
& \left(z_{i-1}+\sum_{j=1}^{r-1} a_{i-1, j} y_{j}\right)\left(z_{i+1}+\sum_{j=1}^{r-1} a_{i+1, j} y_{j}\right)-\left(z_{i}+\sum_{j=1}^{r-1} a_{i j} y_{j}\right)^{2}-y_{i} \sum_{k=0}^{r} p_{i k} z_{k}= \\
= & z_{i-1} z_{i+1}-z_{i}^{2}-\sum_{j=1}^{r-1} a_{i-1, j} z_{i+1} y_{j}-\sum_{j=1}^{r-1} a_{i+1, j} z_{i-1} y_{j}+2 \sum_{j=1}^{r-1} a_{i j} z_{i} y_{j}-\sum_{k=0}^{r} p_{i k} z_{k} y_{i}=^{(*)} \\
= & z_{i-1} z_{i+1}-z_{i}^{2}-\sum_{j=1}^{r-1} a_{i-1, j} z_{i+j+2-r} y_{r-1}-\sum_{j=1}^{r-1} a_{i+1, j} z_{i+j-r} y_{r-1}+ \\
& +2 \sum_{j=1}^{r-1} a_{i j} z_{i+j+1-r} y_{r-1}-\sum_{k=0}^{r} p_{i k} z_{k+i+1-r} y_{r-1},
\end{aligned}
$$

where, in $(*)$, we use the fact that $z_{i} y_{j}-z_{i-1} y_{j+1} \in J$ and the convention that we can use $z_{x} y_{r-1}$ with $x<0$ to mean $z_{0} y_{r+x-1}$.

Hence, we get the following linear equations in the $a_{i j}$ 's:

$$
a_{i-1, h-1}-2 a_{i h}+a_{i+1, h+1}-p_{i h}=0
$$

for $h=0, \ldots, r$ where we assume that $a_{i j}=0$ if $j \leq 0$ or $j \geq r$, for whatever $i$.

With analogous computations, if we apply the change of coordinates to $x_{i} x_{j+1}-x_{i+1} x_{j}-$ $y_{i+1} p_{j}-\cdots-y_{j} p_{i+1}$ we get the following linear equations

$$
a_{i, m-j-2+r}+a_{j+1, m-i-1+r}-a_{j, m-i-2+r}-a_{i+1, m-j-1+r}-\sum_{t=i+1}^{j} p_{i+j+1-t, m-t-1+r}=0 .
$$

It is an easy computation to show that those last equations depend linearly from the previous ones. For example, if we subtract from the last equation the one among (7) we 
get setting $i=j, h=m-i-2+r$, we have the relation

$$
a_{i, m-j-2+r}+a_{j, m-i-2+r}-a_{j-1, m-i-3+r}-a_{i+1, m-j-1+r}-\sum_{t=i+2}^{j} p_{i+j+1-t, m-t-1+r}=0,
$$

which is again of the same form, but with smaller difference between the first subscripts. By iterating, we get that all of them linearly depend from the equations (7).

Now, we prove that the linear system (77) has one solution. To this aim, we collect the equation according to the difference $i-h$ of the subscripts of the variables involved. In fact, notice that in each equation, the difference is constant.

At first, assume that the difference is $i-h=0$. Then, we get the following linear system

$$
\left\{\begin{array}{l}
-2 a_{11}+a_{22}=p_{11} \\
a_{11}-2 a_{22}+a_{33}=p_{22} \\
\vdots \\
a_{i-1,1-i}-2 a_{i i}+a_{i+1, i+1}=p_{i i} \\
\vdots \\
a_{r-2, r-2}-2 a_{r-1, r-1}=p_{r-1, r-1}
\end{array}\right.
$$

The coefficient matrix $M_{r-1}=\left(m_{i j}\right)$ has entries equal to

$$
m_{i j}=\left\{\begin{array}{cl}
-2 & \text { if } i=j \\
1 & \text { if }|i-j|=1 \\
0 & \text { otherwise }
\end{array}\right.
$$

and its determinant is equal to $2 p+1$ if $r-1=2 p$, or to $-2 p$ if $r-1=2 p-1$. In fact, by the Laplace formula,

$$
\operatorname{det}\left(M_{r-1}\right)=-2 \operatorname{det}\left(M_{r-2}\right)-\operatorname{det}\left(M_{r-3}\right),
$$

by direct computation $\operatorname{det}\left(M_{1}\right)=-2, \operatorname{det}\left(M_{2}\right)=3$ and the claim can be easily proved by induction. Hence, the previous linear system has one solution, by Cramer's rule.

Assume now that the difference is equal to $i-h=k>0$. Hence, the corresponding linear system is

$$
\left\{\begin{array}{l}
a_{k+1,1}=p_{k 0} \\
-2 a_{k+1,1}+a_{k+2,2}=p_{k+1,1} \\
a_{k+1,1}-2 a_{k+2,2}+a_{k+3,3}=p_{k+2,2} \\
\vdots \\
a_{r-2, r-2-k}-2 a_{r-1, r-1-k}+a_{r, r-k}=p_{r-2, r-2-k}
\end{array}\right.
$$

and it has one solution for every $k$.

Analogously, the system with $i-h=k<0$ has one solution for every $k$ and the claim follows.

Remark 4.8. Let $X$ be the double structure on a rational normal curve defined in the proof of Theorem 4.6. There is a natural map $\Psi: \operatorname{Aut}\left(\mathbb{P}^{2 r-1}\right) \rightarrow H(2 r, 1,2 r-1)$ defined as $\Psi(g)=g(X)$ where $g(X)$ is the double structure we get by applying $g$ to $X$. Previous Theorem 4.7 is equivalent to ker $\Psi=A u t(C)$. In fact, every automorphism $g$ of $C$ extends to an automorphism $g^{\prime}$ of $L$ that fixes $C . g^{\prime}$ can be further extended to an automorphism 
$g^{\prime \prime}$ of $\mathbb{P}^{2 r-1}$ that fixes $L$. For such a $g^{\prime \prime}$ we have that $g^{\prime \prime}(X)=X$. Hence, $A u t(C) \subseteq \operatorname{ker} \Psi$. By a dimension count, we get that $\Psi$ is surjective if, and only if, ker $\Psi=\operatorname{Aut}(C)$.

Now, we apply the previous results to Gorenstein liaison.

Corollary 4.9. A rational normal curve $C \subseteq L \cong \mathbb{P}^{r} \subseteq \mathbb{P}^{n}$ of degree $r$ is self-linked if, and only if, either $n=r$ or $n=2 r-1$.

Proof. $C$ is self-linked if, and only if, there exists a double structure supported on $C$ that is arithmetically Gorenstein. The claim is then a direct consequence of Theorems 4.4 and 4.6 .

\section{Double CONICS}

In this section, we prove that the general double structure of genus $g \leq-2$ supported on a smooth conic is a smooth point in its Hilbert scheme. Moreover, they are the general element of an irreducible component in the same range of the arithmetic genus. On the other hand, if such double structures are contained in $\mathbb{P}^{3}$, and their genus satisfies $g \geq-1$, then we identify the general element of the irreducible component containing the considered double structures. To achieve the result, we compare the dimension of the family of the double structures of fixed genus with the dimension of $H^{0}\left(X, \mathcal{N}_{X}\right)$, global sections of the normal sheaf of a suitable double conic $X$. In fact, it is well known that $H^{0}\left(X, \mathcal{N}_{X}\right)$ can be identified with the tangent space to the Hilbert scheme at $X$. To get the desired results, we consider first a suitable double conic $X \subset \mathbb{P}^{3}$. We describe its ideal $I_{X}$ and the minimal free resolution

$$
0 \rightarrow F_{3} \rightarrow F_{2} \rightarrow F_{1} \rightarrow I_{X} \rightarrow 0
$$

where $F_{i}$ is a free $R=K[x, y, z, w]$-module, then the $R$-module structure of the global sections of $H_{*}^{0}\left(X, \mathcal{O}_{X}\right)=\bigoplus_{j \in \mathbb{Z}} H^{0}\left(X, \mathcal{O}_{X}(j)\right)$ and finally we compute $H^{0}\left(X, \mathcal{N}_{X}\right)$ as the degree 0 elements of

$$
\operatorname{ker}\left(\operatorname{Hom}\left(F_{1}, H_{*}^{0}\left(X, \mathcal{O}_{X}\right)\right) \rightarrow \operatorname{Hom}\left(F_{2}, H_{*}^{0}\left(X, \mathcal{O}_{X}\right)\right)\right) .
$$

The result for a general double conic $X \subset \mathbb{P}^{n}$ follows from the smoothness of the Hilbert scheme at a degenerate double conic. Furthermore, in $\mathbb{P}^{3}$, we distinguish the case $g(X)$ odd from the case $g(X)$ even, because the ideals have a different minimal number of generators, and so their minimal free resolutions have not comparable free modules and maps. Of course, even if there are differences, we use the same arguments in both cases.

5.1. Case $g$ odd, i.e. $a=2 b$. In this subsection, we will use the following running notation. We set $R=K[x, y, z, w]$, and $C \subset \mathbb{P}^{3}=\operatorname{Proj}(R)$ is the conic defined by the ideal $I_{C}=\left(x z-y^{2}, w\right)$. Let $j: \mathbb{P}^{1} \rightarrow C$ be the isomorphism defined as $j(t: u)=\left(t^{2}: t u\right.$ : $\left.u^{2}: 0\right)$. Finally, we set $\mu: \mathcal{O}_{\mathbb{P}^{1}}(-4) \oplus \mathcal{O}_{\mathbb{P}^{1}}(-2) \rightarrow \mathcal{O}_{\mathbb{P}^{1}}(-4+2 b)$ to be the map defined as $\mu=\left(u^{2 b}, t^{2 b-2}\right)$.

Proposition 5.1. If $X$ is the doubling of $C$ associated to $\mu$, then

(1) $I_{X}=\left\langle w^{2}, w\left(x z-y^{2}\right),\left(x z-y^{2}\right)^{2}, x^{b-1}\left(x z-y^{2}\right)-z^{b} w\right\rangle$;

(2) $w^{2}, w\left(x z-y^{2}\right),\left(x z-y^{2}\right)^{2}, x^{b-1}\left(x z-y^{2}\right)-z^{b} w$ is a Gröbner basis of $I_{X}$ with respect to the reverse lexicographic order; 
(3) the minimal free resolution of $I_{X}$ is

$$
\begin{aligned}
& R(-4) \quad R(-2) \\
& \oplus \oplus
\end{aligned}
$$

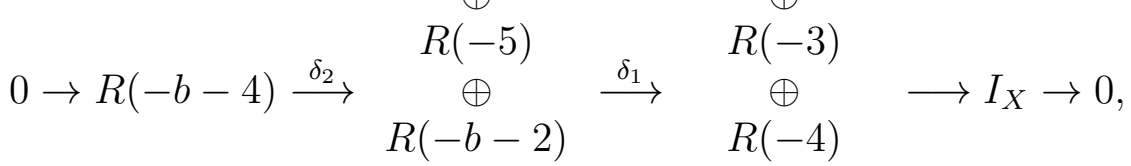

$$
\begin{aligned}
& \begin{array}{cc}
\oplus & \oplus \\
R(-b-3) & R(-b-1)
\end{array}
\end{aligned}
$$

where the maps $\delta_{1}$ and $\delta_{2}$ will be described in the proof.

Proof. The syzygies of $H_{*}^{0}\left(j_{*} \mu\right)$ are generated by $M=\left(\begin{array}{c}x^{b-1} \\ -z^{b}\end{array}\right)$ and so the saturated ideal $I_{X}$ of $X$ is generated by $I_{C}^{2}+\left[I_{C}\right] M$, that is to say,

$$
I_{X}=\left\langle w^{2}, w\left(x z-y^{2}\right),\left(x z-y^{2}\right)^{2}, x^{b-1}\left(x z-y^{2}\right)-z^{b} w\right\rangle .
$$

The generators are a Gröbner basis of $I_{X}$ because their $S$-polynomials reduces to 0 via themselves ([9], Theorem 15.8). Moreover, the free $R$-module $F_{1}$ follows. Let $\left(g_{1}, \ldots, g_{4}\right)$ be a syzygy of $I_{X}$. Then, in $R$, we have

$$
w^{2} g_{1}+w\left(x z-y^{2}\right) g_{2}+\left(x z-y^{2}\right)^{2} g_{3}+x^{b-1}\left(x z-y^{2}\right) g_{4}-z^{b} w g_{4}=0,
$$

that can be rewritten as $w\left(w g_{1}+\left(x z-y^{2}\right) g_{2}-z^{b} g_{4}\right)+\left(x z-y^{2}\right)\left(\left(x z-y^{2}\right) g_{3}+x^{b-1} g_{4}\right)=0$. The two polynomials $w, x z-y^{2}$ are a regular sequence, and so there exists $g \in R$ such that

$$
\begin{aligned}
& w g+\left(x z-y^{2}\right) g_{3}+x^{b-1} g_{4}=0 \\
& w g_{1}+\left(x z-y^{2}\right)\left(g_{2}-g\right)-z^{b} g_{4}=0 .
\end{aligned}
$$

Both $w, x z-y^{2}, x^{b-1}$ and $w, x z-y^{2}, z^{b}$ form a regular sequence, and so we have

$$
\left(\begin{array}{c}
g \\
g_{3} \\
g_{4}
\end{array}\right)=\left(\begin{array}{ccc}
0 & x^{b-1} & -\left(x z-y^{2}\right) \\
-x^{b-1} & 0 & w \\
x z-y^{2} & -w & 0
\end{array}\right)\left(\begin{array}{c}
f_{1} \\
f_{2} \\
f_{3}
\end{array}\right)
$$

and

$$
\left(\begin{array}{c}
g_{1} \\
g_{2}-g \\
-g_{4}
\end{array}\right)=\left(\begin{array}{ccc}
0 & z^{b} & -\left(x z-y^{2}\right) \\
-z^{b} & 0 & w \\
x z-y^{2} & -w & 0
\end{array}\right)\left(\begin{array}{c}
f_{4} \\
f_{5} \\
f_{6}
\end{array}\right)
$$

By comparing the value of $g_{4}$ from the two expressions above, we get the equation $(x z-$ $\left.y^{2}\right)\left(f_{1}+f_{4}\right)=w\left(f_{2}+f_{5}\right)$. By using the same argument as before, there exists $h \in R$ such that $f_{4}=-f_{1}+w h, f_{5}=-f_{2}+\left(x z-y^{2}\right) h$. Hence, it holds

$$
\left(\begin{array}{c}
g_{1} \\
g_{2} \\
g_{3} \\
g_{4}
\end{array}\right)=\left(\begin{array}{cccc}
-\left(x z-y^{2}\right) & 0 & -z^{b} & 0 \\
w & -\left(x z-y^{2}\right) & x^{b-1} & z^{b} \\
0 & w & 0 & -x^{b-1} \\
0 & 0 & -w & x z-y^{2}
\end{array}\right)\left(\begin{array}{c}
f_{6}-z^{b} h \\
f_{3} \\
f_{2} \\
f_{1}
\end{array}\right)
$$

and the $4 \times 4$ matrix represents the map $\delta_{1}$. Of course, the free $R$-module $F_{2}$ follows from $F_{1}$ and from the degrees of the entries of the map $\delta_{1}$. 
The second syzygies of $I_{X}$ can be computed as the first ones, and we get

$$
\delta_{2}=\left(\begin{array}{c}
-z^{b} \\
x^{b-1} \\
x z-y^{2} \\
-w
\end{array}\right)
$$

The last statement of next Proposition is due to the anonymous referee that we thank once more.

Proposition 5.2. $X$ has genus $g(X)=3-2 b$, and the Hartshorne-Rao function of $X$ is

$$
h^{1} \mathcal{I}_{X}(j)=\left\{\begin{array}{cl}
2(j+b)-3 & \text { if }-b+2 \leq j \leq 0 \\
2 b-2 & \text { if } j=1 \\
2(b-j)+1 & \text { if } 2 \leq j \leq b \\
0 & \text { otherwise. }
\end{array}\right.
$$

Moreover, the Hartshorne-Rao module of $X$ is isomorphic to

$$
R /\left\langle w, x z-y^{2}, x^{b-1}, z^{b}\right\rangle(-b+2) .
$$

Proof. The genus and the Hartshorne-Rao function can be computed by using results from section 3 .

For the last statement, we first remark that double conics are minimal curves in the sense of [22]. Otherwise, a double conic would be bilinked down to a degree two curve. But the Hartshorne-Rao function of a degree two curve increases by at most one, and so we can exclude the case. For minimal curves, the map $\delta_{2}^{\vee}$ begins a minimal free resolution of $H_{*}^{1} \mathcal{I}_{X}$ and so the claim follows because the entries of $\delta_{2}$ are a regular sequence.

Now, we can compute the dimension of the degree $d$ global sections of the structure sheaf of $X$. In fact, it holds

Proposition 5.3. $h^{0}\left(X, \mathcal{O}_{X}(d)\right)=4 d+2 b-2$ if $d \geq 2$.

Proof. The short exact sequence

$$
0 \rightarrow \frac{R}{I_{X}} \rightarrow H_{*}^{0}\left(X, \mathcal{O}_{X}\right) \rightarrow H_{*}^{1} \mathcal{I}_{X} \rightarrow 0
$$

allows us to prove the result.

Now, we describe the elements of $H^{0}\left(X, \mathcal{O}_{X}(d)\right)$ for every $d \geq 2$. To start, we can easily describe the elements of $H^{0}\left(X, \mathcal{O}_{X}(d)\right)$ for $d \geq b+1$, because, in the considered range, we have $\left(R / I_{X}\right)_{d}=H^{0}\left(X, \mathcal{O}_{X}(d)\right)$, and so it holds

Proposition 5.4. Let $d \geq b+1$. Then, $H^{0}\left(X, \mathcal{O}_{X}(d)\right)=V_{d}$ where

$$
V_{d}=\left\{p_{1}+y p_{2}+\left(x z-y^{2}\right) p_{3}+\left(x z-y^{2}\right) y p_{4}+w x^{d-b} p_{5}+w x^{d-b-1} y p_{6} \mid p_{i} \in K[x, z]\right\}
$$

and the degrees of the $p_{i}^{\prime}$ s are fixed in such a way that the elements in $V_{d}$ are homogeneous of degree $d$. 
Proof. The generators of $I_{X}$ are a Gröbner basis and so the initial ideal in $\left(I_{X}\right)$ of $I_{X}$ with respect to the reverse lexicographic order is generated by $w^{2}, y^{2} w, y^{4}, x^{b-1} y^{2}$. Hence, the elements in $V_{d}$ are in normal form with respect to $I_{X}$ and so they are linearly independent.

To describe the elements of $H^{0}\left(X, \mathcal{O}_{X}(d)\right)$ for $2 \leq d \leq b$, we first define a suitable global section $\xi$ of degree $-b+2$, and then we compute all the global sections by using $\xi$ and the elements in $\left(R / I_{X}\right)_{d}$.

Definition 5.5. Let $\xi \in H^{0}\left(X, \mathcal{O}_{X}(-b+2)\right)$ be the global section of $X$ defined as

$$
\xi=\frac{w}{x^{b-1}}=\frac{x z-y^{2}}{z^{b}} .
$$

The global section $\xi$ is well defined because for no closed point on $C$ both $x$ and $z$ can be equal to 0 , and because the two descriptions agree on the overlap (in fact $\left.x^{b-1}\left(x z-y^{2}\right)-z^{b} w \in I_{X}\right)$.

Proposition 5.6. $\xi$ verifies the following equalities

(1) $w \xi=\left(x z-y^{2}\right) \xi=0$;

(2) $x^{b-1} \xi=w$;

(3) $z^{b} \xi=x z-y^{2}$.

Proof. They follow easily from the definition of $\xi$ and from the knowledge of the ideal $I_{X}$

Proposition 5.7. Let $2 \leq d \leq b$. Then, $H^{0}\left(X, \mathcal{O}_{X}(d)\right)=V_{d}$ where

$$
\begin{aligned}
V_{d}= & \left\{p_{1}+y p_{2}+\left(x z-y^{2}\right) p_{3}+\left(x z-y^{2}\right) y p_{4}+w p_{5}+w y p_{6}+\right. \\
& \left.\xi\left(x^{d-1} z^{d} q_{1}+x^{d-2} y z^{d-1} q_{2}\right) \mid p_{i} \in K[x, z], q_{j} \in K[x, z]\right\}
\end{aligned}
$$

and the elements in $V_{d}$ are homogeneous of degree $d$.

Proof. Of course, $V_{d} \subseteq H^{0}\left(X, \mathcal{O}_{X}(d)\right)$.

As before, the elements $p_{1}+y p_{2}+\left(x z-y^{2}\right) p_{3}+\left(x z-y^{2}\right) y p_{4}+w p_{5}+w y p_{6}$ are in normal form with respect to $I_{X}$ and so they are linearly independent. Let $\pi: H_{*}^{0} \mathcal{O}_{X} \rightarrow H_{*}^{1} \mathcal{I}_{X}$. It is evident that $\xi \notin \operatorname{ker}(\pi)=R / I_{X}$ and so $\pi(\xi) \neq 0$. Hence $\pi\left(\xi\left(x^{d-1} z^{d} q_{1}+x^{d-2} y z^{d-1} q_{2}\right)\right)=$ $\pi(\xi)\left(x^{d-1} z^{d} q_{1}+x^{d-2} y z^{d-1} q_{2}\right) \in R /\left\langle w, x z-y^{2}, x^{b-1}, z^{b}\right\rangle(-b+2)$. The generators of $\langle w, x z-$ $\left.y^{2}, x^{b-1}, z^{b}\right\rangle$ are a Gröbner basis and $x^{d-1} z^{d} q_{1}+x^{d-2} y z^{d-1} q_{2}$ are in normal form with respect to the given Gröbner basis. Hence, they are linearly independent, and so $V_{d}$ has dimension $\operatorname{dim} V_{d}=4 d+2 b-2$, and the equality $H^{0}\left(X, \mathcal{O}_{X}(d)\right)=V_{d}$ holds.

Now, we compute the degree 0 global sections of $H^{0}\left(X, \mathcal{N}_{X}\right)$ as

$$
H^{0}\left(X, \mathcal{N}_{X}\right)=\operatorname{ker}\left(\operatorname{Hom}\left(F_{1}, H_{*}^{0}\left(X, \mathcal{O}_{X}\right)\right) \stackrel{\delta_{1}^{\vee}}{\longrightarrow} \operatorname{Hom}\left(F_{2}, H^{0}\left(X, \mathcal{O}_{X}\right)\right)\right)_{0},
$$

where, if $\varphi \in \operatorname{Hom}\left(F_{1}, H_{*}^{0}\left(X, \mathcal{O}_{X}\right)\right)$ then $\delta_{1}^{\vee}(\varphi)=\varphi \circ \delta_{1}$. 
Let $F_{1}=\oplus_{i=1}^{4} R e_{i}$ with $\operatorname{deg}\left(e_{1}\right)=2, \operatorname{deg}\left(e_{2}\right)=3, \operatorname{deg}\left(e_{3}\right)=4, \operatorname{deg}\left(e_{4}\right)=b+1$, and assume $b \geq 4$. $\varphi \in \operatorname{ker}\left(\delta_{1}^{\vee}\right)$ if, and only if, the following system is satisfied:

$$
\left\{\begin{array}{l}
\left(x z-y^{2}\right) \varphi\left(e_{1}\right)-w \varphi\left(e_{2}\right)=0 \\
\left(x z-y^{2}\right) \varphi\left(e_{2}\right)-w \varphi\left(e_{3}\right)=0 \\
z^{b} \varphi\left(e_{1}\right)-x^{b-1} \varphi\left(e_{2}\right)+w \varphi\left(e_{4}\right)=0 \\
z^{b} \varphi\left(e_{2}\right)-x^{b-1} \varphi\left(e_{3}\right)+\left(x z-y^{2}\right) \varphi\left(e_{4}\right)=0 .
\end{array}\right.
$$

with $\varphi\left(e_{i}\right) \in H^{0}\left(X, \mathcal{O}_{X}\left(\operatorname{deg}\left(e_{i}\right)\right)\right)$.

To solve the system, we set

- $\varphi\left(e_{1}\right)=p_{11}+y p_{12}+\left(x z-y^{2}\right) p_{13}+w p_{15}+w y p_{16}+\xi\left(x z^{2} q_{11}+y z q_{12}\right)$ with $p_{1 i} \in$ $K[x, z], q_{1 j} \in K[x, z]$ and $\operatorname{deg}\left(p_{11}\right)=2, \operatorname{deg}\left(p_{12}\right)=\operatorname{deg}\left(p_{15}\right)=1, \operatorname{deg}\left(p_{13}\right)=$ $\operatorname{deg}\left(p_{16}\right)=0, \operatorname{deg}\left(q_{11}\right)=b-3, \operatorname{deg}\left(q_{12}\right)=b-2$

- $\varphi\left(e_{2}\right)=p_{21}+y p_{22}+\left(x z-y^{2}\right) p_{23}+\left(x z-y^{2}\right) y p_{24}+w p_{25}+w y p_{26}+\xi\left(x^{2} z^{3} q_{21}+\right.$ $\left.x y z^{2} q_{22}\right)$ with $p_{2 i} \in K[x, z], q_{2 j} \in K[x, z]$ and $\operatorname{deg}\left(p_{21}\right)=3, \operatorname{deg}\left(p_{22}\right)=\operatorname{deg}\left(p_{25}\right)=$ $2, \operatorname{deg}\left(p_{23}\right)=\operatorname{deg}\left(p_{26}\right)=1, \operatorname{deg}\left(p_{24}\right)=0, \operatorname{deg}\left(q_{21}\right)=b-4, \operatorname{deg}\left(q_{22}\right)=b-3 ;$

- $\varphi\left(e_{3}\right)=p_{31}+y p_{32}+\left(x z-y^{2}\right) p_{33}+\left(x z-y^{2}\right) y p_{34}+w p_{35}+w y p_{36}+\xi\left(x^{3} z^{4} q_{31}+\right.$ $\left.x^{2} y z^{3} q_{32}\right)$ with $p_{3 i} \in K[x, z], q_{3 j} \in K[x, z]$ and $\operatorname{deg}\left(p_{31}\right)=4, \operatorname{deg}\left(p_{32}\right)=\operatorname{deg}\left(p_{35}\right)=$ $3, \operatorname{deg}\left(p_{33}\right)=\operatorname{deg}\left(p_{36}\right)=2, \operatorname{deg}\left(p_{34}\right)=1, \operatorname{deg}\left(q_{31}\right)=b-5, \operatorname{deg}\left(q_{32}\right)=b-4 ;$

- $\varphi\left(e_{4}\right)=p_{41}+y p_{42}+\left(x z-y^{2}\right) p_{43}+\left(x z-y^{2}\right) y p_{44}+w x p_{45}+w y p_{46}$ with $p_{4 i} \in$ $K[x, z]$ and $\operatorname{deg}\left(p_{41}\right)=b+1, \operatorname{deg}\left(p_{42}\right)=b, \operatorname{deg}\left(p_{45}\right)=\operatorname{deg}\left(p_{43}\right)=\operatorname{deg}\left(p_{46}\right)=$ $b-1, \operatorname{deg}\left(p_{44}\right)=b-2$.

Theorem 5.8. With the notation as above, $h^{0}\left(X, \mathcal{N}_{X}\right)=7+4 b=13-2 g$, where $g$ is the arithmetic genus of $X$.

Proof. To get the claim, we have to compute the elements in $H^{0}\left(X, \mathcal{N}_{X}\right)$.

Claim: $\varphi \in H^{0}\left(X, \mathcal{N}_{X}\right)$ if, and only if, $\varphi\left(e_{1}\right)=2 w P_{1}+2 w y P_{2}, \varphi\left(e_{2}\right)=\left(x z-y^{2}\right) P_{1}+$ $\left(x z-y^{2}\right) y P_{2}+w P_{3}+w y P_{4}, \varphi\left(e_{3}\right)=2\left(x z-y^{2}\right) P_{3}+2\left(x z-y^{2}\right) y P_{4}, \varphi\left(e_{4}\right)=\left(x^{b-1} P_{3}-\right.$ $\left.z^{b} P_{1}\right)+y\left(x^{b-1} P_{4}-z^{b} P_{2}\right)+\left(x z-y^{2}\right) P_{5}+\left(x z-y^{2}\right) y P_{6}+w x P_{7}+w y P_{8}$ with $P_{i} \in K[x, z]$ for every $i$ and $\operatorname{deg}\left(P_{1}\right)=\operatorname{deg}\left(P_{4}\right)=1, \operatorname{deg}\left(P_{2}\right)=0, \operatorname{deg}\left(P_{3}\right)=2, \operatorname{deg}\left(P_{5}\right)=\operatorname{deg}\left(P_{7}\right)=$ $\operatorname{deg}\left(P_{8}\right)=b-1, \operatorname{deg}\left(P_{6}\right)=b-2$.

If the claim holds, then we get the dimension of $H^{0}\left(X, \mathcal{N}_{X}\right)$ with an easy parameter count. Hence, we prove the Claim.

It is easy to check that if $\varphi$ satisfies the given conditions, then $\varphi \in H^{0}\left(X, \mathcal{N}_{X}\right)$. Conversely, we solve the equations of the system (8) one at a time.

The first equation becomes

$$
\left(x z-y^{2}\right)\left(p_{11}+y p_{12}\right)-w\left(p_{21}+y p_{22}\right)=0 .
$$

As a $R / I_{X}-$ module, the first syzygy module of $\left(x z-y^{2},-w\right)$ is generated by

$$
\left(\begin{array}{ccccc}
x z-y^{2} & w & 0 & 0 & x^{b-1} \\
0 & 0 & w & x z-y^{2} & z^{b}
\end{array}\right)
$$

and so we get the two equations

$$
p_{11}+y p_{12}=0 \quad p_{21}+y p_{22}=0
$$

because $p_{i j} \in K[x, z]$ and by degree argument. 
Again as $R / I_{X}$-module, the first syzygy module of $(1, y)$ is generated by

$$
\left(\begin{array}{c}
y \\
-1
\end{array}\right)
$$

and so the solutions of two equations are $p_{11}=p_{12}=p_{21}=p_{22}=0$.

The second equation, after substituting the computed solutions of the first one, becomes

$$
w\left(p_{31}+y p_{32}\right)=0 .
$$

We have that $p_{3 i} \in K[x, z]$ and so $p_{31}+y p_{32}=0$. Because of the same argument, and the knowledge of the first syzygy module of $(1, y)$, we get that the solutions of this equation are $p_{31}=p_{32}=0$.

The third equation of the system (8) becomes

$$
\begin{aligned}
\left(x z-y^{2}\right) & \left(x^{b-1}\left(p_{15}-p_{23}\right)+x^{b-1} y\left(p_{16}-p_{24}\right)+z^{b} p_{13}+x z^{2} q_{11}+y z q_{12}\right) \\
& -w\left(x^{b-1} p_{25}+x^{b-1} y p_{26}-p_{41}-y p_{42}+x^{2} z^{3} q_{21}+x y z^{2} q_{22}\right)=0 .
\end{aligned}
$$

Because of the knowledge of the first syzygy module of $\left(x z-y^{2},-w\right)$, from the previous equation we get the two ones

$$
x^{b-1}\left(p_{15}-p_{23}\right)+x^{b-1} y\left(p_{16}-p_{24}\right)+z^{b} p_{13}+x z^{2} q_{11}+y z q_{12}=x^{b-1}\left(r_{1}+y r_{2}\right)
$$

and

$$
x^{b-1} p_{25}+x^{b-1} y p_{26}-p_{41}-y p_{42}+x^{2} z^{3} q_{21}+x y z^{2} q_{22}=z^{b}\left(r_{1}+y r_{2}\right)
$$

where $r_{1} \in K[x, z]_{1}, r_{2} \in K[x, z]_{0}$.

The first one can be rewritten as

$$
x^{b-1}\left(p_{15}-p_{23}-r_{1}\right)+z^{b} p_{13}+x z^{2} q_{11}+y\left(x^{b-1}\left(p_{16}-p_{24}-r_{2}\right)+z q_{12}\right)=0 .
$$

From the knowledge of the first syzygy module of $(1, y)$ we get

$$
x^{b-1}\left(p_{15}-p_{23}-r_{1}\right)+z^{b} p_{13}+x z^{2} q_{11}=0
$$

and

$$
x^{b-1}\left(p_{16}-p_{24}-r_{2}\right)+z q_{12}=0 .
$$

Hence, $p_{15}=p_{23}+r_{1}, p_{13}=0, q_{11}=0, p_{16}=p_{24}+r_{2}, q_{12}=0$.

The second equation can be rewritten as

$$
x^{b-1} p_{25}-p_{41}+x^{2} z^{3} q_{21}-z^{b} r_{1}+y\left(-p_{42}+x^{b-1} p_{26}+x z^{2} q_{22}-z^{b} r_{2}\right)=0 .
$$

By using the same argument as before, we get

$$
x^{b-1} p_{25}-p_{41}+x^{2} z^{3} q_{21}-z^{b} r_{1}=0
$$

and

$$
-p_{42}+x^{b-1} p_{26}+x z^{2} q_{22}-z^{b} r_{2}=0 .
$$

Hence, $p_{41}=x^{b-1} p_{25}+x^{2} z^{3} q_{21}-z^{b} r_{1}, p_{42}=x^{b-1} p_{26}+x z^{2} q_{22}-z^{b} r_{2}$.

The last equation of the system (8) becomes

$$
\begin{gathered}
\left(x z-y^{2}\right)\left(z^{b}\left(p_{23}-r_{1}\right)+2 x^{2} z^{3} q_{21}+y\left(z^{b}\left(p_{24}-r_{2}\right)+2 x z^{2} q_{22}\right)\right)- \\
-w\left(z^{b}\left(p_{33}-2 p_{25}\right)+x^{b-1} p_{35}+x^{3} z^{4} q_{31}+y\left(z^{b}\left(p_{34}-2 p_{26}\right)+x^{b-1} p_{36}+x^{2} z^{3} q_{32}\right)\right)=0 .
\end{gathered}
$$


Then, there exist $r_{3} \in K[x, z]_{2}, r_{4} \in K[x, z]_{1}$ such that the two following equalities hold

$$
z^{b}\left(p_{23}-r_{1}\right)+2 x^{2} z^{3} q_{21}+y\left(z^{b}\left(p_{24}-r_{2}\right)+2 x z^{2} q_{22}\right)=x^{b-1}\left(r_{3}+y r_{4}\right)
$$

and

$$
z^{b}\left(p_{33}-2 p_{25}\right)+x^{b-1} p_{35}+x^{3} z^{4} q_{31}+y\left(z^{b}\left(p_{34}-2 p_{26}\right)+x^{b-1} p_{36}+x^{2} z^{3} q_{32}\right)=z^{b}\left(r_{3}+y r_{4}\right) .
$$

From the first equation, we get

$$
z^{b}\left(p_{23}-r_{1}\right)-x^{b-1} r_{3}+2 x^{2} z^{3} q_{21}=0
$$

and

$$
z^{b}\left(p_{24}-r_{2}\right)-x^{b-1} r_{4}+2 x z^{2} q_{22}=0 .
$$

Hence, $r_{1}=p_{23}, r_{2}=p_{24}, r_{3}=r_{4}=0, q_{21}=q_{22}=0$. From the second equation, we get

$$
z^{b}\left(p_{33}-2 p_{25}\right)+x^{b-1} p_{35}+x^{3} z^{4} q_{31}=0
$$

and

$$
z^{b}\left(p_{34}-2 p_{26}\right)+x^{b-1} p_{36}+x^{2} z^{3} q_{32}=0 .
$$

As before, we deduce that $p_{33}=2 p_{25}, p_{34}=2 p_{26}, p_{35}=p_{36}=0, q_{31}=q_{32}=0$.

Summarizing, we obtain the following:

(1) $\varphi\left(e_{1}\right)=2 w\left(p_{23}+y p_{24}\right)$,

(2) $\varphi\left(e_{2}\right)=\left(x z-y^{2}\right)\left(p_{23}+y p_{24}\right)+w\left(p_{25}+y p_{26}\right)$;

(3) $\varphi\left(e_{3}\right)=2\left(x z-y^{2}\right)\left(p_{25}+y p_{26}\right)$,

(4) $\varphi\left(e_{4}\right)=x^{b-1}\left(p_{25}+y p_{26}\right)-z^{b}\left(p_{23}+y p_{24}\right)+\left(x z-y^{2}\right)\left(p_{43}+y p_{44}\right)+w\left(x p_{45}+y p_{46}\right)$, and the claim follows with the obvious substitutions.

Remark 5.9. We computed $h^{0}\left(X, \mathcal{N}_{X}\right)$ by using the function <normal_sheaf of the computer algebra software Macaulay (see [2]), in the cases $0 \leq b \leq 3$.

If $b=3$, or equivalently $g=-3$, we get that the dimension of the degree 0 global sections of the normal sheaf of $X$ with saturated ideal

$$
I_{X}=\left\langle w^{2}, w\left(x z-y^{2}\right),\left(x z-y^{2}\right)^{2}, x^{2}\left(x z-y^{2}\right)-z^{3} w\right\rangle
$$

is equal to $h^{0}\left(X, \mathcal{N}_{X}\right)=19=13-2 g$.

If $b=2$, i.e. $g=-1$, the dimension of the degree 0 global sections of the normal sheaf of the double conic $X$ defined by the ideal

$$
I_{X}=\left\langle w^{2}, w\left(x z-y^{2}\right),\left(x z-y^{2}\right)^{2}, x\left(x z-y^{2}\right)-z^{2} w\right\rangle
$$

is equal to $h^{0}\left(X, \mathcal{N}_{X}\right)=16 \neq 13-2 g$.

If $b=1$, i.e. $g=1$, the double conic $X$ is defined by the ideal

$$
I_{X}=\left\langle w^{2}, w\left(x z-y^{2}\right),\left(x z-y^{2}\right)^{2},\left(x z-y^{2}\right)-z w\right\rangle
$$

and $h^{0}\left(X, \mathcal{N}_{X}\right)=16 \neq 13-2 g$.

At last, if $b=0$, i.e. $g=3$, then the double conic $X$ is defined by the ideal

$$
I_{X}=\left\langle w,\left(x z-y^{2}\right)^{2}\right\rangle
$$

and $h^{0}\left(X, \mathcal{N}_{X}\right)=17 \neq 13-2 g$. 
5.2. Case $g$ even, i.e. $a=2 b+1$. In this subsection, we repeat what we did in the previous subsection, by sketching the main differences.

The running notation of the subsection are the following. As before, we set $R=$ $K[x, y, z, w]$ and $C \subset \mathbb{P}^{3}=\operatorname{Proj}(R)$ is the conic defined by the ideal $I_{C}=\left(x z-y^{2}, w\right)$. $C$ is isomorphic to $\mathbb{P}^{1}$ via $j: \mathbb{P}^{1} \rightarrow C$ defined as $j(t: u)=\left(t^{2}: t u: u^{2}: 0\right)$. We set $\mu: \mathcal{O}_{\mathbb{P}^{1}}(-4) \oplus \mathcal{O}_{\mathbb{P}^{1}}(-2) \rightarrow \mathcal{O}_{\mathbb{P}^{1}}(-3+2 b)$ defined as $\mu=\left(u^{2 b+1}, t^{2 b-1}\right)$.

Proposition 5.10. If $X$ is the doubling of $C$ associated to $\mu$, then

(1) $I_{X}=\left\langle w^{2}, w\left(x z-y^{2}\right),\left(x z-y^{2}\right)^{2}, x^{b}\left(x z-y^{2}\right)-y z^{b} w, x^{b-1} y\left(x z-y^{2}\right)-z^{b+1} w\right\rangle$;

(2) the generators of $I_{X}$ are a Gröbner basis with respect to the reverse lexicographic order;

(3) the minimal free resolution of $I_{X}$ is

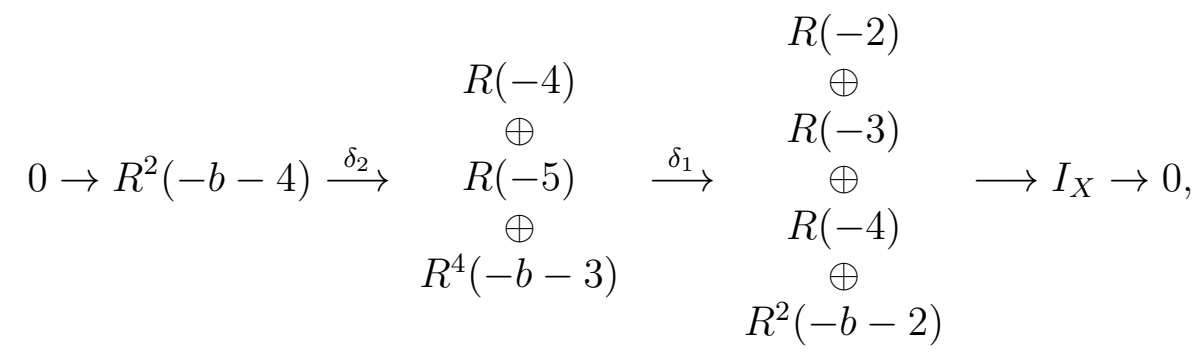

where the maps $\delta_{1}$ and $\delta_{2}$ will be described in the proof.

Proof. The first syzygy module of $H_{*}^{0}\left(j_{*} \mu\right)=\left(y z^{b}, x^{b}\right)$ is generated by

$$
N=\left(\begin{array}{cc}
x^{b} & x^{b-1} y \\
-y z^{b} & -z^{b+1}
\end{array}\right)
$$

Let $M$ be the matrix we get by reading $N$ over $R$ and not over $R / I_{C}$. Then, the saturated ideal of $X$ is

$$
I_{X}=\left\langle w^{2}, w\left(x z-y^{2}\right),\left(x z-y^{2}\right)^{2}, x^{b}\left(x z-y^{2}\right)-y z^{b} w, x^{b-1} y\left(x z-y^{2}\right)-z^{b+1} w\right\rangle .
$$

The check on $S$-polynomials holds on the generators of $I_{X}$ and so they are a Gröbner basis.

To compute the first syzygy module of $I_{X}$ we proceed as in the proof of Proposition 5.1. The computation is quite similar and uses the same ideas. Hence, we write only the maps $\delta_{1}$ and $\delta_{2}$ :

$$
\delta_{1}=\left(\begin{array}{cccccc}
x z-y^{2} & 0 & -y z^{b} & 0 & 0 & z^{b+1} \\
-w & x z-y^{2} & x^{b} & z^{b} & 0 & -x^{b-1} y \\
0 & -w & 0 & 0 & -x^{b-1} & 0 \\
0 & 0 & -w & -y & z & 0 \\
0 & 0 & 0 & x & -y & w
\end{array}\right)
$$


and

$$
\delta_{2}=\left(\begin{array}{cc}
z^{b} & 0 \\
0 & x^{b-1} \\
-y & -z \\
w & 0 \\
0 & -w \\
-x & -y
\end{array}\right)
$$

Proposition 5.11. $X$ has genus $g(X)=2-2 b$, the Hartshorne-Rao function of $X$ is equal to

$$
h^{1} \mathcal{I}_{X}(j)=\left\{\begin{array}{cl}
2(b+j-1) & \text { if }-b+1 \leq j \leq 0 \\
2 b-1 & \text { if } j=1 \\
2(b-j+1) & \text { if } 2 \leq j \leq b+1 \\
0 & \text { otherwise }
\end{array}\right.
$$

and $\delta_{2}^{\vee}$ is a presentation matrix for $H_{*}^{1} \mathcal{I}_{X}$.

Proof. The proof of the first two statements rests on results from Section 3. The last statement follows from the minimality of $X$ in its biliaison class.

Proposition 5.12. $h^{0}\left(X, \mathcal{O}_{X}(d)\right)=4 d+2 b-1$ for $d \geq 2$.

Proof. See Proposition 5.3 .

As before, we describe the elements of $H^{0}\left(X, \mathcal{O}_{X}(d)\right)$ for every $d \geq 2$. At first, we describe the elements of $H^{0}\left(X, \mathcal{O}_{X}(d)\right)$ for $d \geq b+1$, because, in the considered range, we have $\left(R / I_{X}\right)_{d}=H^{0}\left(X, \mathcal{O}_{X}(d)\right)$, and so it holds

Proposition 5.13. Let $d \geq b+1$. Then, $H^{0}\left(X, \mathcal{O}_{X}(d)\right)$ is equal to

$$
\left\{p_{1}+y p_{2}+\left(x z-y^{2}\right) p_{3}+\left(x z-y^{2}\right) y p_{4}+w x^{d-b-1} p_{5}+w x^{d-b-1} y p_{6} \mid p_{i} \in K[x, z]\right\}
$$

where the degrees of the $p_{i}^{\prime} s$ are fixed in such a way that the elements are homogeneous of degree $d$.

To describe the elements of $H^{0}\left(X, \mathcal{O}_{X}(d)\right)$ for $2 \leq d \leq b$, this time we need two suitable global sections $\xi_{1}, \xi_{2}$ of degree $-b+2$, and then we compute all the global sections by using $\xi_{1}, \xi_{2}$ and the elements in $\left(R / I_{X}\right)_{d}$.

Definition 5.14. Let $\xi_{1}, \xi_{2} \in H^{0}\left(X, \mathcal{O}_{X}(-b+2)\right)$ be the global section of $X$ defined as

$$
\xi_{1}=\frac{x z-y^{2}}{z^{b}}=\frac{y w}{x^{b}} \text { and } \xi_{2}=\frac{y\left(x z-y^{2}\right)}{z^{b+1}}=\frac{w}{x^{b-1}} .
$$

The global sections $\xi_{1}$ and $\xi_{2}$ are well defined because for no closed point on $C$ both $x$ and $z$ can be equal to 0 , and because the two definitions agree on the overlap (see the last two generators of $I_{X}$.)

Proposition 5.15. $\xi_{1}$ and $\xi_{2}$ verify the following equalities

(1) $w \xi_{1}=\left(x z-y^{2}\right) \xi_{1}=w \xi_{2}=\left(x z-y^{2}\right) \xi_{2}=0$;

(2) $x^{b} \xi_{1}=y w, z^{b} \xi_{1}=x z-y^{2}$; 
(3) $x^{b-1} \xi_{2}=w, y z^{b} \xi_{2}=x\left(x z-y^{2}\right), z^{b+1} \xi_{2}=y\left(x z-y^{2}\right)$;

(4) $x \xi_{1}=y \xi_{2}, y \xi_{1}=z \xi_{2}$.

Proof. The equalities easily follow from the definition of $\xi_{1}, \xi_{2}$ and from the knowledge of the ideal $I_{X}$.

Proposition 5.16. Let $2 \leq d \leq b$. Then $H^{0}\left(X, \mathcal{O}_{X}(d)\right)=V_{d}$ where

$$
\begin{aligned}
V_{d}= & \left\{p_{1}+y p_{2}+\left(x z-y^{2}\right) p_{3}+\left(x z-y^{2}\right) y p_{4}+w p_{5}+w y p_{6}+\right. \\
& \left.x^{d-2} z^{d-1} \xi_{2}\left(z q_{1}+y q_{2}\right) \mid p_{i} \in K[x, z], q_{j} \in K[x, z]\right\}
\end{aligned}
$$

and the elements in $V_{d}$ are homogeneous of degree $d$.

Proof. We can apply the same argument as in Proposition 5.7, with the only difference that

$$
\left\{f \in R \mid f \xi_{2} \in R / I_{X}\right\}=\left\langle w, x z-y^{2}, x^{b-1}, y z^{b}, z^{b+1}\right\rangle
$$

as can be easily computed via computer algebra techniques.

Remark 5.17. Thanks to the relations of previous Proposition 5.15, we can write the elements in $H^{0}\left(X, \mathcal{O}_{X}(d)\right)$ without using $\xi_{1}$. Analogously, one can write them using $\xi_{1}$ but not $\xi_{2}$. The choice of using only one between $\xi_{1}, \xi_{2}$ allows to simplify the following computations.

We want to compute the degree 0 global sections of the normal sheaf of $X$ as

$$
H^{0}\left(X, \mathcal{N}_{X}\right)=\operatorname{ker}\left(\operatorname{Hom}\left(F_{1}, H_{*}^{0}\left(X, \mathcal{O}_{X}\right)\right) \stackrel{\delta_{1}^{\vee}}{\longrightarrow} \operatorname{Hom}\left(F_{2}, H_{*}^{0}\left(X, \mathcal{O}_{X}\right)\right)\right)_{0}
$$

where $F_{1}=R(-2) \oplus R(-3) \oplus R(-4) \oplus R^{2}(-b-2)$ and $F_{2}=R(-4) \oplus R(-5) \oplus R^{4}(-b-3)$ and $\delta_{1}^{\vee}$ is the dual of $\delta_{1}: F_{2} \rightarrow F_{1}$.

To this end, let $\varphi \in \operatorname{Hom}\left(F_{1}, H_{*}^{0}\left(X, \mathcal{O}_{X}\right)\right)$ be a degree 0 map. Then, if $F_{1}=\oplus_{i=1}^{5} R e_{i}$ with $\operatorname{deg}\left(e_{1}\right)=2, \operatorname{deg}\left(e_{2}\right)=3, \operatorname{deg}\left(e_{3}\right)=4, \operatorname{deg}\left(e_{4}\right)=\operatorname{deg}\left(e_{5}\right)=b+2$, we have that $\varphi\left(e_{i}\right) \in H^{0}\left(X, \mathcal{O}_{X}\left(\operatorname{deg}\left(e_{i}\right)\right)\right)$, and so, if we assume that $b \geq 4$, we can set

- $\varphi\left(e_{1}\right)=p_{11}+y p_{12}+\left(x z-y^{2}\right) p_{13}+w p_{15}+w y p_{16}+\xi_{2}\left(z^{2} q_{11}+y z q_{12}\right)$, with $\operatorname{deg}\left(p_{11}\right)=$ $2, \operatorname{deg}\left(p_{12}\right)=\operatorname{deg}\left(p_{15}\right)=1, \operatorname{deg}\left(p_{13}\right)=\operatorname{deg}\left(p_{16}\right)=0, \operatorname{deg}\left(q_{11}\right)=\operatorname{deg}\left(q_{12}\right)=b-2 ;$

- $\varphi\left(e_{2}\right)=p_{21}+y p_{22}+\left(x z-y^{2}\right) p_{23}+\left(x z-y^{2}\right) y p_{24}+w p_{25}+w y p_{26}+\xi_{2}\left(x z^{3} q_{21}+x y z^{2} q_{22}\right)$, with $\operatorname{deg}\left(p_{21}\right)=3, \operatorname{deg}\left(p_{22}\right)=\operatorname{deg}\left(p_{25}\right)=2, \operatorname{deg}\left(p_{23}\right)=\operatorname{deg}\left(p_{26}\right)=1, \operatorname{deg}\left(p_{24}\right)=$ $0, \operatorname{deg}\left(q_{21}\right)=\operatorname{deg}\left(q_{22}\right)=b-3$;

- $\varphi\left(e_{3}\right)=p_{31}+y p_{32}+\left(x z-y^{2}\right) p_{33}+\left(x z-y^{2}\right) y p_{34}+w p_{35}+w y p_{36}+\xi_{2}\left(x^{2} z^{4} q_{31}+\right.$ $\left.x^{2} y z^{3} q_{32}\right)$, with $\operatorname{deg}\left(p_{31}\right)=4, \operatorname{deg}\left(p_{32}\right)=\operatorname{deg}\left(p_{35}\right)=3, \operatorname{deg}\left(p_{33}\right)=\operatorname{deg}\left(p_{36}\right)=$ $2, \operatorname{deg}\left(p_{34}\right)=1, \operatorname{deg}\left(q_{31}\right)=\operatorname{deg}\left(q_{32}\right)=b-4$

- $\varphi\left(e_{4}\right)=p_{41}+y p_{42}+\left(x z-y^{2}\right) p_{43}+\left(x z-y^{2}\right) y p_{44}+w x p_{45}+w x y p_{46}$, with $\operatorname{deg}\left(p_{41}\right)=$ $b+2, \operatorname{deg}\left(p_{42}\right)=b+1, \operatorname{deg}\left(p_{43}\right)=\operatorname{deg}\left(p_{45}\right)=b, \operatorname{deg}\left(p_{44}\right)=\operatorname{deg}\left(p_{46}\right)=b-1 ;$

- $\varphi\left(e_{5}\right)=p_{51}+y p_{52}+\left(x z-y^{2}\right) p_{53}+\left(x z-y^{2}\right) y p_{54}+w x p_{55}+w x y p_{56}$, with $\operatorname{deg}\left(p_{51}\right)=$ $b+2, \operatorname{deg}\left(p_{52}\right)=b+1, \operatorname{deg}\left(p_{53}\right)=\operatorname{deg}\left(p_{55}\right)=b, \operatorname{deg}\left(p_{54}\right)=\operatorname{deg}\left(p_{56}\right)=b-1$. 
Of course, $\varphi \in H^{0}\left(X, \mathcal{N}_{X}\right)$ if, and only if, $\delta_{1}^{\vee}(\varphi)=\varphi \circ \delta_{1}=0$, and so we get the following system

$$
\left\{\begin{array}{l}
\left(x z-y^{2}\right) \varphi\left(e_{1}\right)-w \varphi\left(e_{2}\right)=0 \\
\left(x z-y^{2}\right) \varphi\left(e_{2}\right)-w \varphi\left(e_{3}\right)=0 \\
-y z^{b} \varphi\left(e_{1}\right)+x^{b} \varphi\left(e_{2}\right)-w \varphi\left(e_{4}\right)=0 \\
z^{b} \varphi\left(e_{2}\right)-y \varphi\left(e_{4}\right)+x \varphi\left(e_{5}\right)=0 \\
-x^{b-1} \varphi\left(e_{3}\right)+z \varphi\left(e_{4}\right)-y \varphi\left(e_{5}\right)=0 \\
z^{b+1} \varphi\left(e_{1}\right)-x^{b-1} y \varphi\left(e_{2}\right)+w \varphi\left(e_{5}\right)=0 .
\end{array}\right.
$$

A technical result in the computation of $H^{0}\left(X, \mathcal{N}_{X}\right)$ is the knowledge of the generators of two syzygy modules. In particular, it holds

Lemma 5.18. In $R / I_{X}$, the first syzygy module of $\left(x z-y^{2},-w\right)$ is generated by

$$
\left(\begin{array}{cccccc}
x z-y^{2} & w & 0 & 0 & x^{b} & x^{b-1} y \\
0 & 0 & w & x z-y^{2} & y z^{b} & z^{b+1}
\end{array}\right)
$$

while the first syzygy module of $(1, y)$ is minimally generated by

$$
\left(\begin{array}{c}
y \\
-1
\end{array}\right)
$$

The proof is based on standard Gröbner bases arguments.

Thanks to the previous Lemma, we can solve system (13), one equation at a time, and we get

Theorem 5.19. With the notation as above, $h^{0}\left(X, \mathcal{N}_{X}\right)=9+4 b=13-2 g$.

Proof. To prove the statement, we have to compute the elements in $H^{0}\left(X, \mathcal{N}_{X}\right)$.

Claim: $\varphi \in H^{0}\left(X, \mathcal{N}_{X}\right)$ if, and only if, there exist $\alpha, \beta \in K$, and $P_{1}, \ldots, P_{8} \in K[x, z]$, of suitable degrees, such that

- $\varphi\left(e_{1}\right)=2 w\left(P_{1}+y P_{2}\right)$;

- $\varphi\left(e_{2}\right)=\left(x z-y^{2}\right)\left(P_{1}+y P_{2}\right)+w\left(\alpha x^{2}+z P_{3}+y P_{4}\right)$;

- $\varphi\left(e_{3}\right)=2\left(x z-y^{2}\right)\left(\alpha x^{2}+z P_{3}+y P_{4}\right)$;

- $\varphi\left(e_{4}\right)=x^{b}\left(\alpha x^{2}+z P_{3}+y P_{4}\right)-y z^{b}\left(P_{1}+y P_{2}\right)+\left(x z-y^{2}\right)\left(x P_{5}+y P_{6}+x^{b-1} P_{3}\right)+$ $x w\left(x P_{7}+y P_{8}+\beta z^{b}\right)$

- $\varphi\left(e_{5}\right)=x^{b-1} y\left(\alpha x^{2}+z P_{3}+y P_{4}\right)-z^{b+1}\left(P_{1}+y P_{2}\right)+\left(x z-y^{2}\right)\left(y P_{5}+z P_{6}-x^{b-1} P_{4}\right)+$ $x w\left(y P_{7}+z P_{8}-\alpha z^{b}\right)$.

If the claim holds, then we can compute $h^{0}\left(X, \mathcal{N}_{X}\right)$ with an easy parameter count. Hence, we prove the claim. Its proof is a quite long computation where we use the same ideas as in the proof of Theorem 5.8 .

Remark 5.20. Now, we consider the cases not covered by Theorem 5.19, namely $1 \leq$ $b \leq 3$. We consider the double conic defined by the ideal

$$
I_{X}=\left\langle w^{2}, w\left(x z-y^{2}\right),\left(x z-y^{2}\right)^{2}, x^{b}\left(x z-y^{2}\right)-y z^{b} w, x^{b-1} y\left(x z-y^{2}\right)-z^{b+1} w\right\rangle
$$

with $b=1,2,3$, and we compute $h^{0}\left(X, \mathcal{N}_{X}\right)$ by using Macaulay (see [2]).

If $b=3$, we get $h^{0}\left(X, \mathcal{N}_{X}\right)=21=13-2 g$, because $g=2-2 b=-4$.

If $b=2$, we get $h^{0}\left(X, \mathcal{N}_{X}\right)=17=13-2 g$, because $g=-2$.

If $b=1$, then we get $H^{0}\left(X, \mathcal{N}_{X}\right)=16 \neq 13-2 g$, because, in this case, $g=0$. 
5.3. Case $X \subseteq \mathbb{P}^{n}, n \geq 4$. Now, we suppose that $X$ is a suitable double conic in $\mathbb{P}^{n}, n \geq 4$. Theorem 5.21. Let $C \subset \mathbb{P}^{n}$ be the conic defined by the ideal

$$
I_{C}=\left\langle x_{0} x_{2}-x_{1}^{2}, x_{3}, \ldots, x_{n}\right\rangle,
$$

and let $j: \mathbb{P}^{1} \rightarrow C$ be the isomorphism defined as $j(t: u)=\left(t^{2}: t u: u^{2}: 0: \cdots\right.$ : $0)$. Let $\mu: \mathcal{O}_{\mathbb{P} 1}(-4) \oplus \mathcal{O}_{\mathbb{P}^{1}}^{n-2}(-2) \rightarrow \mathcal{O}_{\mathbb{P} 1}(-4+a), a \geq 5$, be the map defined as $\mu=$ $\left(u^{a}, t^{a-2}, 0, \ldots, 0\right)$, and let $X$ be the double structure on $C$ associated to $\mu$ and $j$. Then, $h^{0}\left(X, \mathcal{N}_{X}\right) \leq(n-1)(5-g)+3$.

Proof. $\mathcal{O}_{\mathbb{P 1}}^{n-2}(-2)$ is contained in the kernel of $\mu$. Hence, $x_{3}, \ldots, x_{n} \in I_{X}$ i.e. $X$ is degenerate and it is contained in the linear space $L$ of dimension 3 . Then, we can consider both the normal sheaf $\mathcal{N}_{X, L}$ of $X$ in $L$, and the normal sheaf $\mathcal{N}_{X}$ of $X$ in $\mathbb{P}^{n}$. They are related via the exact sequence

$$
0 \rightarrow \mathcal{N}_{X, L} \longrightarrow \mathcal{N}_{X} \longrightarrow \mathcal{O}_{X}(1)^{n-3} .
$$

In particular, we have the inequality

$$
h^{0}\left(X, \mathcal{N}_{X}\right) \leq h^{0}\left(X, \mathcal{N}_{X, L}\right)+(n-3) h^{0}\left(X, \mathcal{O}_{X}(1)\right) .
$$

By hypothesis, $a \geq 5$ and so the arithmetic genus $g$ of $X$ satisfies $g \leq-2$. By Theorems 5.8, 5.19 and the Remarks after them, $h^{0}\left(X, \mathcal{N}_{X, L}\right)=13-2 g$, while $h^{0}\left(X, \mathcal{O}_{X}(1)\right)=$ $\operatorname{dim}_{K}\left(R / I_{X}\right)_{1}+h^{1} \mathcal{I}_{X}(1)=4+a-2=5-g$ as proved in Propositions 3.9. Then,

$$
h^{0}\left(X, \mathcal{N}_{X}\right) \leq 13-2 g+(n-3)(5-g)=(n-1)(5-g)+3 .
$$

5.4. Remarks on the Hilbert schemes $\mathcal{H i l b}_{4 t+1-g}\left(\mathbb{P}^{n}\right)$. In this last subsection, we use the previous results to get information on the irreducible components containing the double structures of genus $g$ on conics.

Theorem 5.22. If $g \leq-2$, then $\overline{H(4, g, n)}$ is a generically smooth irreducible component of $\mathcal{H} i b_{4 t+1-g}\left(\mathbb{P}^{n}\right)$ of dimension $(n-1)(5-g)+3$.

Proof. As proved in Corollary 2.14, every irreducible component containing $\overline{H(4, g, n)}$ has dimension greater than or equal to $(n-1)(5-g)+3$, but the tangent space to the Hilbert scheme $\mathcal{H i l b}_{4 t+1-g}\left(\mathbb{P}^{n}\right)$ at the double conic described in Theorem 5.21 has dimension lesser than or equal to $(n-1)(5-g)+3$. Hence, the point corresponding to the double conic considered in Theorem $[5.21$ is smooth, $\overline{H(4, g, n)}$ is irreducible of dimension $\operatorname{dim} \overline{H(4, g, n)}=(n-1)(5-g)+3$ and the point corresponding to the double conic considered in Theorem 5.21 is smooth, i.e. $\overline{H(4, g, n)}$ is generically smooth.

Now, we add some remarks to $\overline{H(4, g, 3)}$ for $g \geq-1$.

Proposition 5.23. If $g=-1$, the general element of $\overline{H(4,-1,3)}$ is the union of two smooth conics without common points, and a double structure on a smooth conic is a smooth point of $\overline{H(4,-1,3)}$. 
Proof. By a simple parameter count, the family of two disjoint conics has dimension 16, which is equal to the dimension of the tangent space to $\overline{H(4,-1,3)}$ at the double conic considered in Theorem 5.8 and in the subsequent Remark. The claim follows if we exhibit a family whose general element is a disjoint union of two conics, and whose special fiber is the considered double structure. The ideal

$$
\left\langle w, x z-y^{2}\right\rangle \cap\left\langle w+t x, t z^{2}+x z-y^{2}\right\rangle \subseteq K[x, y, z, w, t]
$$

gives a flat family over $\mathbb{A}^{1}$ with the required properties.

Proposition 5.24. If $g=0$, the general element of $\overline{H(4,0,3)}$ is a rational quartic curve, and a double structure on a smooth conic is a smooth point of $\overline{H(4,0,3)}$.

Proof. By a simple parameter count, the family of the rational quartic curves has dimension 16 , which is equal to the dimension of the tangent space to $\overline{H(4,0,3)}$ at the double conic considered in Theorem 5.19 and in the subsequent Remark. The claim follows if we exhibit a family whose general element is a rational quartic curve, and whose special fiber is the considered double structure. The ideal

$$
\left\langle w^{2}+t(x y-z w), y\left(x z-y^{2}\right)-z^{2} w\right\rangle:\left\langle x y-z w, y^{2}, y w, w^{2}\right\rangle \subseteq K[x, y, z, w, t]
$$

gives a flat family over $\mathbb{A}^{1}$ with the required properties. In fact, a general quartic curve is linked to two skew lines or to a double line of genus -1 via a complete intersection of type $(2,3)$. The general element of the family is the residual intersection of a double line of genus -1 on a smooth quadric surface, while the special element is the residual intersection to the same double line on a double plane.

Proposition 5.25. If $g=1$, the general element of $\overline{H(4,1,3)}$ is the complete intersection of two quadric surfaces, and a double structure on a smooth conic is a smooth point of $\overline{H(4,1,3)}$.

Proof. By a simple parameter count, the family of the complete intersections of two quadric surfaces has dimension 16 , which is equal to the dimension of the tangent space to $\overline{H(4,1,3)}$ at the double conic considered in Theorem 5.8 and in the subsequent Remark. The claim follows because it is easy to check that the ideal of the considered double conic is the complete intersection of $w^{2}$ and $x z-y^{2}-z w$.

Proposition 5.26. If $g=3$, the general element of $\overline{H(4,3,3)}$ is a plane quartic curve, and a double structure on a smooth conic is a smooth point of $\overline{H(4,3,3)}$.

Proof. By a simple parameter count, the family of the plane quartic curves has dimension 17 , which is equal to the dimension of the tangent space to $\overline{H(4,3,3)}$ at the double conic considered in Theorem 5.8 and in the subsequent Remark. The claim follows because the double conic we considered is a plane quartic curve.

\section{REFERENCES}

[1] E. Ballico, G. Bolondi, J.C. Migliore, The Lazarsfeld-Rao problem for liaison classes of twocodimensional subschemes of $\mathbb{P}^{n}$, Amer. J. Math. 113 (1991), no. 1, 117-128. 
[2] D. Bayer, M. Stillmann, Macaulay: a system for computation in algebraic geometry and commutative algebra, Source and object code available for Unix and Macintosh computers. Contact the authors, or download from zariski.harvard.edu via anonymous ftp. (login: anonymous, password: any, cd Macaulay), Cambridge U.P., 1982-1990.

[3] D. Bayer, D. Eisenbud, Ribbons and their canonical embeddings, Trans. Amer. Math. Soc. 347 (1995), no. 3, 719-756.

[4] C. Banica, O. Forster, Multiplicity structures on space curves, Contemporary Math. 58 (1986), 47-64.

[5] C. Banica, N. Manolache, Rank 2 stable vector bundles on $\mathbb{P}^{3}(\mathbb{C})$ with Chern classes $c_{1}=-1, c_{2}=4$, Math. Z. 190 (1985), 315-339.

[6] M. Boratyński, S. Greco, When does an ideal arise from the Ferrand construction?, Boll. Un. Mat. Ital. B (7) 1 (1987), no. 1, 247-258.

[7] M. Boratyński, S. Greco,Hilbert functions and Betti numbers in a flat family, Ann. Mat. Pura Appl. (4) 142 (1985), 277-292 (1986).

[8] M. Casanellas, R.M. Miró-Roig, On the Lazarsfeld-Rao property for Gorenstein liaison classes, J. Pure and Appl. Alg. 179 (2003), 7-12.

[9] D. Eisenbud, Commutative algebra, GTM 150, Springer-Verlag, 2004.

[10] D. Eisenbud, M. Green, Clifford indices of ribbons, Trans. Amer. Math. Soc. 347 (1995), no. 3, $757-765$.

[11] D. Ferrand, Courbes gauches et fibres de rang 2, C.R. Acad. Sci. Paris Ser. A 281 (1977), 345-347.

[12] L.Y. Fong, Rational ribbons and deformation of hyperelliptic curves, J. Algebraic Geom. 2 (1993), no. 2, 295-307.

[13] F. Gaeta, Nuove ricerche sulle curve sghembe algebriche di risiduale finito e sui gruppi di punti del piano, (French) An. Mat. Pura Appl. (4) 31, (1950), 1-64.

[14] A. Grothendieck, Techniques de construction et théorèmes d' existence en géométrie algébrique. IV. Le schémas de Hilbert, Séminaire Bourbaki, Exp. No. 221, vol. 8, Soc.Math.France, Paris, 1995, pp.249-276.

[15] R. Hartshorne, Connectedness of the Hilbert scheme, Publ. Math. de I.H.E.S. 29 (1966), pp. 261-304.

[16] R. Hartshorne, Some examples of Gorenstein liaison in codimension three, Collect. Math. 53 (2002), $21-48$.

[17] R. Hartshorne, Algebraic Geometry, GTM 52, Springer-Verlag, 1977.

[18] R. Hartshorne, E. Schlesinger, Curves in the double plane, Special issue in honor of Robin Hartshorne, Comm. Algebra 28 (2000), no. 12, 5655-5676.

[19] G.R. Kempf, Algebraic varieties, London Mathematical Society Lecture Note Series, 172, Cambridge University Press, Cambridge, 1993.

[20] N. Manolache, Multiple structures on smooth support, Math. Nachr. 167 (1994), 157-202.

[21] N. Manolache, Double rational normal curves with linear syzygies, Manuscripta Math. 104 (2001), no. 4, 503-517.

[22] M. Martin-Deschamps, D. Perrin, Sur la classification des courbes gauches, Astérisque 184-185, Soc. Math. de France (1990).

[23] J.C. Migliore, Introduction to liaison theory and deficiency modules, Progress in Mathematics 165 Birkhäuser, 1998.

[24] U. Nagel, R. Notari, M.L. Spreafico, On the even Gorenstein liaison classes of ropes on a line, Dedicated to Silvio Greco on the occasion of his 60th birthday (Catania, 2001). Matematiche (Catania) 55 (2000), no. 2, 483-498 (2002).

[25] U. Nagel, R. Notari, M.L. Spreafico, Curves of degree two and ropes on a line: their ideals and even liaison classes, J. Algebra 265 (2003), no. 2, 772-793.

[26] U. Nagel, R. Notari, M.L. Spreafico, The Hilbert scheme of degree two curves and certain ropes, Internat. J. Math. 17 (2006), no. 7, 835-867.

[27] S. Nollet, E. Schlesinger, Hilbert schemes of degree four curves, Compositio Math. 139 (2003), no. 2, 169-196. 
[28] E. Sernesi, Deformations of algebraic schemes, Grundlehren der Mathematischen Wissenschaften 334, Springer-Verlag, Berlin, 2006.

[29] J. Wahl, On cohomology of the square of an ideal sheaf, J. Algebraic Geom. 6 (1997), no. 3, 481-511.

Dipartimento di Matematica, Politecnico di Milano, I-20133 Milano, Italy

E-mail address: roberto.notari@polimi.it

Departamento de Matemáticas, Universidad de Extremadura, E-06071 Badajoz, España. E-mail address: ojedamc@unex.es

Dipartimento di Matematica, Politecnico di Torino, I-10129 Torino, Italy

E-mail address: maria.spreafico@polito.it 